MATHEMATICS OF COMPUTATION

Volume 68, Number 225, January 1999, Pages 25-53

S 0025-5718(99)01035-2

\title{
CONVERGENCE \\ OF NONCONFORMING MULTIGRID METHODS WITHOUT FULL ELLIPTIC REGULARITY
}

\author{
SUSANNE C. BRENNER
}

\begin{abstract}
We consider nonconforming multigrid methods for symmetric positive definite second and fourth order elliptic boundary value problems which do not have full elliptic regularity. We prove that there is a bound $(<1)$ for the contraction number of the $W$-cycle algorithm which is independent of mesh level, provided that the number of smoothing steps is sufficiently large. We also show that the symmetric variable $V$-cycle algorithm is an optimal preconditioner.
\end{abstract}

\section{INTRODUCTION}

The multigrid theory for conforming finite element methods where the finite element spaces on successive grids are nested is now well understood (cf., for example, the books [42], [46], [10] and the references therein).

However, for certain problems the simplest finite element methods are nonconforming or conforming but nonnested. For example, the simplest method for the stationary Stokes equations uses the Crouzeix-Raviart element (nonconforming), and the simplest finite element methods for the plate bending problem use the Morley finite element (nonconforming) or the (reduced) Hsieh-Clough-Tocher macroelement (conforming but nonnested). Also, some simple nonconforming methods can overcome the phenomenon of locking in elasticity problems and plate problems (cf., [5], [37], [28], [67]).

The convergence of multigrid methods for nonconforming elements was studied in [14]-[18], [20], [21], [23], [49], [9], [43], [50], [62], [63], [52], [55], [64], [65], [68] and [60]. The convergence of the multigrid method for macro-elements was studied in [66]. The results for the nonconforming or conforming but nonnested multigrid methods can also be obtained from the more abstract theory of Bramble, Pasciak and Xu (cf., [13]) once their "regularity and approximation" assumption is verified for each concrete problem. The results in all the papers (except [65]; see below) cited above for nonconforming and macro elements have been obtained under the condition that the underlying boundary value problem has full elliptic regularity.

Received by the editor April 13, 1995.

1991 Mathematics Subject Classification. Primary 65N55, 65N30.

Key words and phrases. Multigrid methods, nonconforming finite elements, macro elements, preconditioner, $W$-cycle, variable $V$-cycle.

This work was supported in part by the National Science Foundation under Grant No. DMS94-96275.

(C)1999 American Mathematical Society 
In this paper we study the convergence of multigrid methods for nonconforming finite elements without assuming full elliptic regularity. We follow the methodology of Bank and Dupont in [6], where the convergence of conforming, nested $W$-cycle multigrid methods is established without full elliptic regularity. The two key ingredients in their approach are: (i) the equivalence between mesh-dependent norms and fractional order Sobolev norms on the finite element space, and (ii) a duality argument involving fractional order Sobolev spaces. Since the nonconforming finite element space may not be a subspace of the fractional order Sobolev space, there are no straightforward generalizations of (i) and (ii) to the nonconforming case. We overcome this difficulty by relating the nonconforming finite element to a conforming finite element.

The idea of using conforming "relatives" in the treatment of nonconforming finite elements was first used in the context of additive Schwarz preconditioners for nonconforming finite elements (cf., [22], [24], [25]). Let $(K, \mathcal{P}, \mathcal{N})$ and $(K, \tilde{\mathcal{P}}, \tilde{\mathcal{N}})$ be two finite elements (cf., [30], [27]), where $K$ is the shared element domain, $\mathcal{P}$ and $\tilde{\mathcal{P}}$ are the spaces of shape functions, and $\mathcal{N}$ and $\tilde{\mathcal{N}}$ are the sets of nodal variables. We say that $(K, \mathcal{P}, \mathcal{N}) \preceq(K, \tilde{\mathcal{P}}, \tilde{\mathcal{N}})$ if $\mathcal{P} \subseteq \tilde{\mathcal{P}}$ and $\mathcal{N} \subseteq \tilde{\mathcal{N}}$, and refer to $(K, \tilde{\mathcal{P}}, \tilde{\mathcal{N}})$ as a "relative" of $(K, \mathcal{P}, \mathcal{N})$. Let $V$ and $\tilde{V}$ be the finite element spaces on the same triangulation associated with $(K, \mathcal{P}, \mathcal{N})$ and $(K, \tilde{\mathcal{P}}, \tilde{\mathcal{N}})$ respectively. Then we say that $V \preceq \tilde{V}$ if $(K, \mathcal{P}, \mathcal{N}) \preceq(K, \tilde{\mathcal{P}}, \tilde{\mathcal{N}})$. Our idea is to find a conforming finite element space $\tilde{V}_{h}$ for a given nonconforming finite element space $V_{h}$ such that $V_{h} \preceq \tilde{V}_{h}$. Then we obtain multigrid convergence results for $V_{h}$ by exploiting its connection with $\tilde{V}_{h}$. In the theory we do not require that the $\tilde{V}_{h}$ on successive grids be nested. Therefore, by applying the theory to $V_{h}=\tilde{V}_{h}$, we also have multigrid convergence results for conforming but nonnested finite element methods.

After the completion of the first draft of this paper, we learned that $W$-cycle convergence in the (nonconforming) energy norm without full elliptic regularity was obtained in [65] by a different technique. However, one of the assumptions (Assumption A.4) in [65] concerns a discretization error estimate for nonconforming finite elements which is not in the literature and was not proved in [65]. It turns out that this estimate follows from our theory (cf., the remark after Theorem 3.8). Thus the estimate from our approach combined with the theory in [65] would give another complete proof of the $W$-cycle convergence in the (nonconforming) energy norm.

The rest of the paper is organized as follows. In Section 2 we set up the notation and assumptions of an abstract framework for our finite element multigrid analysis which is applicable to both second and fourth order problems. Preliminary estimates are established in Section 3. In Section 4 we obtain the convergence of the $k$-th level $W$-cycle algorithm and the full multigrid $W$-cycle method in both the (nonconforming) energy norm and a lower order norm. In particular we show that the contraction number of the $k$-th level $W$-cycle algorithm is bounded away from 1 uniformly when the number of smoothing steps is sufficiently large. For fourth order problems, the convergence in the lower order norm and the connection to the conforming relative result in a better pointwise convergence rate for the nonconforming method. We also prove that the symmetric variable $V$-cycle multigrid algorithm is an optimal preconditioner. Applications of our theory to second and fourth order problems are given in Sections 5 and 6. 
For future reference, we state the $W$-cycle and variable $V$-cycle algorithms here. Let $V_{1}, V_{2}, \ldots$ be finite-dimensional vector spaces, and let $A_{k}: V_{k} \longrightarrow V_{k}, I_{k-1}^{k}$ : $V_{k-1} \longrightarrow V_{k}$ and $I_{k}^{k-1}: V_{k} \longrightarrow V_{k-1}$. The equation to be solved is

$$
A_{k} z=g \text {. }
$$

The $W$-cycle multigrid algorithm. Let $m_{1}$ and $m_{2}$ be two nonnegative integers. The $W$-cycle multigrid algorithm with initial guess $z_{0}$ yields $W M G\left(k, z_{0}, g\right)$ as an approximate solution to the equation (1.1).

For $k=1, W M G\left(1, z_{0}, g\right)$ is the solution obtained from a direct method. In other words,

$$
W M G\left(1, z_{0}, g\right)=A_{1}^{-1} g .
$$

For $k>1, W M G\left(k, z_{0}, g\right)$ is defined recursively in three steps.

- Pre-smoothing. Let $z_{l} \in V_{k}\left(1 \leq l \leq m_{1}\right)$ be defined recursively by the equations

$$
z_{l}=z_{l-1}+\frac{1}{\Lambda_{k}}\left(g-A_{k} z_{l-1}\right), \quad 1 \leq l \leq m_{1},
$$

where $\Lambda_{k}$ dominates the spectral radius of $A_{k}$.

- Correction. Let $\bar{g}:=I_{k}^{k-1}\left(g-A_{k} z_{m_{1}}\right)$. Let $q_{i} \in V_{k-1}(0 \leq i \leq 2)$ be defined recursively by

$$
\begin{aligned}
& q_{0}=0, \quad \text { and } \\
& q_{i}=W M G\left(k-1, q_{i-1}, \bar{g}\right), \quad i=1,2 .
\end{aligned}
$$

Let $z_{m_{1}+1}=z_{m_{1}}+I_{k-1}^{k} q_{2}$.

- Post-smoothing. Let $z_{l} \in V_{k}\left(m_{1}+2 \leq l \leq m_{1}+m_{2}+1\right)$ be defined recursively by the equations

$$
z_{l}=z_{l-1}+\frac{1}{\Lambda_{k}}\left(g-A_{k} z_{l-1}\right), \quad m_{1}+2 \leq l \leq m_{1}+m_{2}+1 .
$$

Then $W M G\left(k, z_{0}, g\right)=z_{m_{1}+m_{2}+1}$.

The symmetric variable $V$-cycle algorithm. Let $m_{j}(j=2, \ldots, k)$ be positive integers which are chosen so that $\beta_{0} m_{j} \leq m_{j-1} \leq \beta_{1} m_{j}$ for $j=3, \ldots, k$, and $1<\beta_{0} \leq \beta_{1}$. The symmetric variable $V$-cycle multigrid algorithm with initial guess $z_{0}$ yields $\mathcal{V} M G\left(k, z_{0}, g\right)$ as an approximate solution to the equation (1.1).

For $k=1, \mathcal{V} M G\left(1, z_{0}, g\right)$ is the solution obtained from a direct method. In other words,

$$
\mathcal{V} M G\left(1, z_{0}, g\right)=A_{1}^{-1} g .
$$

For $k>1, \mathcal{V} M G\left(k, z_{0}, g\right)$ is defined recursively in three steps.

- Pre-smoothing. Let $z_{l} \in V_{k}\left(1 \leq l \leq m_{k}\right)$ be defined recursively by the equations

$$
z_{l}=z_{l-1}+\frac{1}{\Lambda_{k}}\left(g-A_{k} z_{l-1}\right), \quad 1 \leq l \leq m_{k},
$$

where $\Lambda_{k}$ dominates the spectral radius of $A_{k}$.

- Correction. Let $\bar{g}:=I_{k}^{k-1}\left(g-A_{k} z_{m_{k}}\right)$, and

$$
q=\mathcal{V} M G(k-1,0, \bar{g}) .
$$

Let $z_{m_{k}+1}=z_{m_{k}}+I_{k-1}^{k} q$. 
- Post-smoothing. Let $z_{l} \in V_{k}\left(m_{k}+2 \leq l \leq 2 m_{k}+1\right)$ be defined recursively by the equations

$$
z_{l}=z_{l-1}+\frac{1}{\Lambda_{k}}\left(g-A_{k} z_{l-1}\right), \quad m_{k}+2 \leq l \leq 2 m_{k}+1,
$$

Then $\mathcal{V} M G\left(k, z_{0}, g\right)=z_{2 m_{k}+1}$.

\section{An abstract Framework}

In this section we set up an abstract framework for our finite element multigrid analysis, which will be carried out in Sections 3 and 4 under the assumptions stated here. Throughout this paper, $\ell=1$ (second order problems) or 2 (fourth order problems), and $\alpha \in(0,1]$. The case $\alpha=1$ corresponds to the case of full elliptic regularity.

We begin with the continuous problem. Let $V$ be a Hilbert space and $a(\cdot, \cdot)$ be a symmetric bilinear form on $V$ which is bounded and coercive:

$$
\begin{aligned}
\left|a\left(v_{1}, v_{2}\right)\right| & \lesssim\left\|v_{1}\right\|_{V}\left\|v_{2}\right\|_{V} \quad \forall v_{1}, v_{2} \in V, \\
a(v, v) & \gtrsim\|v\|_{V}^{2} \quad \forall v \in V .
\end{aligned}
$$

In order to avoid the proliferation of constants, we adopt the notation $\lesssim, \gtrsim$ and $\approx$. The statement $F \lesssim G$ (or $G \gtrsim F$ ) means that $F$ is bounded by $G$ multiplied by a constant which is independent of mesh sizes. The statement $F \approx G$ means $F \lesssim G$ and $G \lesssim F$.

Let $F \in V^{\prime}$. The continuous problem is to find $u \in V$ such that

$$
a(u, v)=F(v) \quad \forall v \in V .
$$

There exists a unique solution of (2.1) by (B), (C) and the Riesz Representation Theorem.

We assume that there exist two other Hilbert spaces $Z$ and $W$ such that

$$
\begin{aligned}
& Z \hookrightarrow V \hookrightarrow W, \\
& \|u\|_{Z} \lesssim\|F\|_{W^{\prime}},
\end{aligned}
$$

where $F \in W^{\prime}$ and $u$ is the solution to (2.1).

Moreover, we assume the spaces $Z$ and $W$ are related by the following duality estimate.

$$
|a(\zeta, v)| \lesssim\|\zeta\|_{Z}\|v\|_{W} \quad \forall \zeta \in Z, v \in V .
$$

Remark. In applications $V$ is a subspace of $H^{\ell}(\Omega), W$ is a subspace of $H^{\ell-\alpha}(\Omega)$, and $Z$ is a subspace of $H^{\ell+\alpha}(\Omega)$. The elliptic regularity for (2.1) is then given by $(\mathrm{R}-1)$ and $(\mathrm{R}-2)$.

Next we describe the finite element spaces. Let $V_{1}, V_{2}, \ldots$ and $\tilde{V}_{1}, \tilde{V}_{2}, \ldots$ be two sequences of finite-dimensional vector spaces with corresponding mesh parameters $h_{1}, h_{2}, \ldots$ We assume that there exist positive constants $C_{1}$ and $C_{2}$, independent of the mesh sizes, such that

$$
C_{1} h_{k-1} \leq h_{k} \leq C_{2} h_{k-1} \quad \text { and } \quad 0<C_{1} \leq C_{2}<1 .
$$

We assume that the spaces $V_{k}$ and $\tilde{V}_{k}$ are connected to the spaces of the continuous problem through the following relations:

$$
\tilde{V}_{k} \subseteq V \quad \text { (i.e., } \tilde{V}_{k} \text { is conforming), }
$$


and there exists a Hilbert space $X$ such that

$$
\begin{aligned}
& W \hookrightarrow X \text { and } V_{k}, \tilde{V}_{k} \subseteq X \text { for } \quad k \geq 1, \\
& W=[X, V]_{1-(\alpha / \ell)},
\end{aligned}
$$

where $[X, V]_{1-(\alpha / \ell)}$ denotes the interpolation space obtained from $X$ and $V$ by the complex method of interpolation (cf., [7], [61], [44]). When $\alpha=1=\ell$, we interpret $[X, V]_{0}$ to be the space $X$.

Remark. Note that we do not assume $\tilde{V}_{k-1} \subseteq \tilde{V}_{k}$, i.e., $\tilde{V}_{k}$ is conforming but not necessarily nested, and the space $V_{k}$ can be nonconforming and hence nonnested. However, they are all inside the space $X$, which is just $L^{2}(\Omega)$ in applications.

Let $V_{0}=\{0\}$. We assume that, for each positive integer $k$, there exists a symmetric positive definite bilinear form $a_{k}(\cdot, \cdot)$ on $V_{k-1}+V_{k}+V$ such that $a_{k}(\cdot, \cdot)$ reduces to $a(\cdot, \cdot)$ on $V$ and $a_{k-1}(\cdot, \cdot)$ on $V_{k-1}$. The (possibly nonconforming) energy norm $\|\cdot\|_{k}$ on $V_{k-1}+V_{k}+V$ is then defined to be

$$
\|v\|_{k}=\left[a_{k}(v, v)\right]^{1 / 2} .
$$

It follows from the boundedness and coercivity conditions $(\mathrm{B})$ and $(\mathrm{C})$ that

$$
\|v\|_{V} \approx\|v\|_{k} \quad \forall v \in V .
$$

Furthermore, we assume the following inverse estimate holds for $k \geq 1$ :

$$
\|v\|_{k} \lesssim h_{k}^{-\ell}\|v\|_{X} \quad \forall v \in V_{k-1}+V_{k}+\tilde{V}_{k} .
$$

We assume there exists an interpolation operator $\Pi_{k}: V \longrightarrow V_{k}$ which satisfies the following interpolation estimates for $k \geq 1$ :

$$
\begin{aligned}
\left\|\Pi_{k} v-v\right\|_{X}+h_{k}^{\ell}\left\|\Pi_{k} v\right\|_{k} \lesssim h_{k}^{\ell}\|v\|_{V} & \forall v \in V, \\
\left\|\zeta-\Pi_{k} \zeta\right\|_{X}+h_{k}^{\ell}\left\|\zeta-\Pi_{k} \zeta\right\|_{k} \lesssim h_{k}^{\ell+\alpha}\|\zeta\|_{Z} & \forall \zeta \in Z .
\end{aligned}
$$

The spaces $V_{k}$ and $\tilde{V}_{k}$ are connected by the operators $E_{k}: V_{k} \longrightarrow \tilde{V}_{k}$ and $F_{k}: \tilde{V}_{k} \longrightarrow V_{k}$ which satisfy the following:

$$
\begin{array}{rlrl}
\left\|E_{k} v-v\right\|_{X} & \lesssim h_{k}^{\ell}\|v\|_{k} & & \forall v \in V_{k}, \\
\left\|E_{k} \Pi_{k} \zeta-\zeta\right\|_{X}+h_{k}^{\ell}\left\|E_{k} \Pi_{k} \zeta-\zeta\right\|_{V} \lesssim h_{k}^{\ell+\alpha}\|\zeta\|_{Z} & \forall \zeta \in Z, \\
\left\|F_{k} \tilde{v}-\tilde{v}\right\|_{X} \lesssim h_{k}^{\ell}\|\tilde{v}\|_{V} & & \forall \tilde{v} \in \tilde{V}_{k}, \\
F_{k} E_{k} v & =v & & \forall v \in V_{k} .
\end{array}
$$

Remark. In applications the constructions and analyses of $E_{k}$ and $F_{k}$ rely on the relation $V_{k} \preceq \tilde{V}_{k}$.

Let $\zeta \in Z$ and $\zeta_{k} \in V_{k}$ be related by

$$
a\left(\zeta, E_{k} v\right)=a_{k}\left(\zeta_{k}, v\right) \quad \forall v \in V_{k}
$$

We assume that

$$
\begin{aligned}
\left|a_{k}\left(\zeta-\zeta_{k}, v\right)\right| & \lesssim h_{k}^{\alpha}\|\zeta\|_{Z}\|v\|_{k} \quad \forall v \in V_{k}, \zeta \in Z, \\
\left|a_{k}\left(\zeta-\zeta_{k}, \Pi_{k} \xi\right)\right| & \lesssim h_{k}^{2 \alpha}\|\zeta\|_{Z}\|\xi\|_{Z} \quad \forall \zeta, \xi \in Z .
\end{aligned}
$$


Remark. In applications the estimates (N-1) and (N-2) are obtained by modifying standard estimates for nonconforming finite element methods.

So far the relations between the spaces $V_{k}$ have not been specified. Now we connect $V_{k-1}$ and $V_{k}$ by the coarse-to-fine intergrid transfer operator $I_{k-1}^{k}: V_{k-1} \longrightarrow$ $V_{k}$. We assume that the following estimates on $I_{k-1}^{k}$ hold:

$$
\begin{array}{rlrl}
\left\|I_{k-1}^{k} v-v\right\|_{X} & \lesssim h_{k}^{\ell}\|v\|_{k-1} & \forall v \in V_{k-1}, \\
\left\|I_{k-1}^{k} \Pi_{k-1} \zeta-\Pi_{k} \zeta\right\|_{X} & \lesssim h_{k}^{\ell+\alpha}\|\zeta\|_{Z} \quad \forall \zeta \in Z .
\end{array}
$$

We also assume that $V_{k}$ is equipped with the inner product $(\cdot, \cdot)_{k}$ such that

$$
(v, v)_{k} \approx(v, v)_{X} \quad \forall v \in V_{k} .
$$

We can then define $A_{k}: V_{k} \longrightarrow V_{k}$ by

$$
\left(A_{k} v_{1}, v_{2}\right)_{k}=a_{k}\left(v_{1}, v_{2}\right) \quad \forall v_{1}, v_{2} \in V_{k} .
$$

By our assumptions on $a_{k}(\cdot, \cdot), A_{k}$ is a linear symmetric positive definite operator.

It follows from $(\mathrm{I}),(\mathrm{P})$ and $(2.5)$ that

$$
\rho\left(A_{k}\right) \leq C_{*} h_{k}^{-2 \ell},
$$

where $C_{*}>0$ is independent of $k$. The number $\Lambda_{k}$ in (1.2), (1.4), (1.5) and (1.7) is then defined by $\Lambda_{k}=C_{*} h_{k}^{-2 \ell}$.

Finally, the fine-to-coarse intergrid transfer operator $I_{k}^{k-1}: V_{k} \longrightarrow V_{k-1}$ is defined by

$$
\left(I_{k}^{k-1} v_{1}, v_{2}\right)_{k-1}=\left(v_{1}, I_{k-1}^{k} v_{2}\right)_{k} \quad \forall v_{1} \in V_{k} \quad \text { and } \quad v_{2} \in V_{k-1} .
$$

For the convergence analysis we also need the operator $P_{k}^{k-1}: V_{k} \longrightarrow V_{k-1}$ defined by

$$
a_{k-1}\left(P_{k}^{k-1} v_{1}, v_{2}\right)=a_{k}\left(v_{1}, I_{k-1}^{k} v_{2}\right) \quad \forall v_{1} \in V_{k} \quad \text { and } \quad v_{2} \in V_{k-1} .
$$

It is easy to see from (2.5), (2.7) and (2.8) that the operators $A_{k}, A_{k-1}, I_{k}^{k-1}$ and $P_{k}^{k-1}$ are related by

$$
A_{k-1} P_{k}^{k-1}=I_{k}^{k-1} A_{k}
$$

\section{Preliminary estimates}

In this section we derive some estimates in preparation for the convergence analysis in the next section.

Lemma 3.1. The following estimates hold:

$$
\begin{aligned}
& \left\|E_{k} v\right\|_{V} \lesssim\|v\|_{k} \quad \text { and } \quad\left\|E_{k} v\right\|_{X} \lesssim\|v\|_{X} \quad \forall v \in V_{k} \text {, } \\
& \left\|F_{k} \tilde{v}\right\|_{k} \lesssim\|\tilde{v}\|_{V} \quad \text { and } \quad\left\|F_{k} \tilde{v}\right\|_{X} \lesssim\|\tilde{v}\|_{X} \quad \forall v \in \tilde{V}_{k}, \\
& \left\|I_{k-1}^{k} v\right\|_{k} \lesssim\|v\|_{k-1} \quad \text { and } \quad\left\|I_{k-1}^{k} v\right\|_{X} \lesssim\|v\|_{X} \quad \forall v \in V_{k-1} .
\end{aligned}
$$

Proof. Using (I), (2.3) and (E) we have

$$
\begin{aligned}
& \left\|E_{k} v\right\|_{V} \lesssim\left\|E_{k} v-v\right\|_{k}+\|v\|_{k} \lesssim h_{k}^{-\ell}\left\|E_{k} v-v\right\|_{X}+\|v\|_{k} \lesssim\|v\|_{k} \\
& \left\|E_{k} v\right\|_{X} \lesssim\left\|E_{k} v-v\right\|_{X}+\|v\|_{X} \lesssim h_{k}^{\ell}\|v\|_{k}+\|v\|_{X} \lesssim\|v\|_{X} .
\end{aligned}
$$

The estimates (3.2)-(3.3) are similarly established by using (I), (F) and (I-1). 
Lemma 3.2. The following estimates hold:

$$
\begin{array}{rlrl}
\left\|E_{k} \Pi_{k} \zeta-\zeta\right\|_{W} & \lesssim h_{k}^{2 \alpha}\|\zeta\|_{Z} & & \forall \zeta \in Z, \\
\left\|E_{k} \Pi_{k} v-v\right\|_{X}+h_{k}^{\ell}\left\|E_{k} \Pi_{k} v\right\|_{V} \lesssim h_{k}^{\ell}\|v\|_{V} & \forall v \in V .
\end{array}
$$

Proof. The estimate (3.4) follows immediately from (C-3), (EП) and interpolation (cf., [7], [61]). The estimate (3.5) is obtained from (П-1), (E) and (3.1) as follows:

$$
\begin{aligned}
\left\|E_{k} \Pi_{k} v-v\right\|_{X}+h_{k}^{\ell}\left\|E_{k} \Pi_{k} v\right\|_{V} \\
\quad \lesssim\left\|E_{k}\left(\Pi_{k} v-v\right)\right\|_{X}+\left\|E_{k} v-v\right\|_{X}+h_{k}^{\ell}\|v\|_{V} \\
\quad \lesssim\left\|\Pi_{k} v-v\right\|_{X}+h_{k}^{\ell}\|v\|_{V} \lesssim h_{k}^{\ell}\|v\|_{V} .
\end{aligned}
$$

For the convergence analysis, we need the following mesh-dependent norms on $V_{k}$ :

$$
\|v\|_{s, k}^{2}=\left(A_{k}^{s / \ell} v, v\right)_{k} \quad \forall v \in V_{k} .
$$

The spaces $\left(V_{k},\|\cdot\|_{k, s}\right)$ form a Hilbert scale (cf., [44]).

From (2.2), (2.5), (2.6), (3.7), (P) and the Cauchy-Schwarz inequality, we have

$$
\begin{aligned}
\|v\|_{0, k} & =\sqrt{(v, v)_{k}} \approx\|v\|_{X} & & \forall v \in V_{k}, \\
\|v\|_{\ell, k} & =\|v\|_{k} & & \forall v \in V_{k}, \\
\|v\|_{s, k} & \lesssim h_{k}^{t-s}\|v\|_{t, k} & & \forall v \in V_{k} \quad \text { and } t<s, \\
\left|a_{k}\left(v_{1}, v_{2}\right)\right| & \leq\left\|v_{1}\right\|_{\ell+t, k}\left\|v_{2}\right\|_{\ell-t, k} & & \forall v_{1}, v_{2} \in V_{k}, t \in \mathbb{R} .
\end{aligned}
$$

Lemma 3.3. The following estimates hold:

$$
\begin{aligned}
\left\|I_{k-1}^{k} v\right\|_{s, k} & \lesssim\|v\|_{s, k-1} & & \forall 0 \leq s \leq \ell, v \in V_{k-1}, \\
\left\|P_{k}^{k-1} v\right\|_{t, k-1} & \lesssim\|v\|_{t, k} & & \forall \ell \leq t \leq 2 \ell, v \in V_{k}, \\
\left\|I_{k-1}^{k} \Pi_{k-1} \zeta-\Pi_{k} \zeta\right\|_{\ell-\alpha, k} & \lesssim h_{k}^{2 \alpha}\|\zeta\|_{Z} & & \forall \zeta \in Z .
\end{aligned}
$$

Proof. The estimate (3.12) follows from (3.3), (3.8), (3.9) and interpolation (cf., [44]). The estimate (3.13) then follows from (2.8), (3.12) and duality.

From (I-2), (3.8), and (3.10) we have

$$
\begin{array}{ll}
\left\|I_{k-1}^{k} \Pi_{k-1} \zeta-\Pi_{k} \zeta\right\|_{0, k} \lesssim h_{k}^{\ell+\alpha}\|\zeta\|_{Z} & \forall \zeta \in Z, \\
\left\|I_{k-1}^{k} \Pi_{k-1} \zeta-\Pi_{k} \zeta\right\|_{\ell, k} \lesssim h_{k}^{\alpha}\|\zeta\|_{Z} & \forall \zeta \in Z .
\end{array}
$$

The estimate (3.14) follows from (3.15), (3.16) and interpolation.

Lemma 3.4. We have the following equivalence of norms:

$$
\|v\|_{\ell-\alpha, k} \approx\left\|E_{k} v\right\|_{W} \quad \forall v \in V_{k} .
$$

Proof. From (C-3), (3.1), (3.8) and (3.9), we obtain by interpolation that

$$
\left\|E_{k} v\right\|_{W} \lesssim\|v\|_{\ell-\alpha, k} \quad \forall v \in V_{k} .
$$

Let $Q_{k}: X \longrightarrow \tilde{V}_{k}$ be the orthogonal projection with respect to the inner product of $X$. Then from (I), (FE), (П-1), (3.2), (3.5), (3.8) and (3.9) we have

$$
\begin{array}{rlrl}
\left\|F_{k} Q_{k} v\right\|_{0, k} & \lesssim\left\|F_{k} Q_{k} v\right\|_{X} \lesssim\left\|Q_{k} v\right\|_{X} \lesssim\|v\|_{X} & & \forall v \in X, \\
\left\|F_{k} Q_{k} v\right\|_{\ell, k} & \lesssim\left\|F_{k} Q_{k}\left(v-E_{k} \Pi_{k} v\right)\right\|_{\ell, k}+\left\|\Pi_{k} v\right\|_{\ell, k} & & \\
& \lesssim h_{k}^{-\ell}\left\|v-E_{k} \Pi_{k} v\right\|_{X}+\|v\|_{V} \lesssim\|v\|_{V} & \forall v \in V .
\end{array}
$$


We find by (C-3), (3.19), (3.20) and interpolation that

$$
\left\|F_{k} Q_{k} v\right\|_{\ell-\alpha, k} \lesssim\|v\|_{W} \quad \forall v \in W .
$$

In particular, we have by (FE) and (3.21) that

$$
\|v\|_{\ell-\alpha, k}=\left\|F_{k} Q_{k} E_{k} v\right\|_{\ell-\alpha, k} \lesssim\left\|E_{k} v\right\|_{W} \quad \forall v \in V_{k} .
$$

Theorem 3.5. Let $\zeta \in Z$ and $\zeta_{k} \in V_{k}$ be related by (2.4). Then the following estimates hold:

$$
\begin{aligned}
\left\|\zeta-\zeta_{k}\right\|_{k} & \lesssim h_{k}^{\alpha}\|\zeta\|_{Z}, \\
\left\|\Pi_{k} \zeta-\zeta_{k}\right\|_{\ell-\alpha, k} & \lesssim h_{k}^{2 \alpha}\|\zeta\|_{Z} .
\end{aligned}
$$

Proof. We have the following estimate for nonconforming methods (cf., [27]):

$$
\left\|\zeta-\zeta_{k}\right\|_{k} \leq \inf _{v \in V_{k}}\|\zeta-v\|_{k}+\sup _{v \in V_{k} \backslash\{0\}} \frac{\left|a_{k}\left(\zeta-\zeta_{k}, v\right)\right|}{\|v\|_{k}} .
$$

The estimate (3.23) follows from (N-1), (П-2) and (3.25).

By (3.17) and duality we have

$$
\left\|\Pi_{k} \zeta-\zeta_{k}\right\|_{\ell-\alpha, k} \approx\left\|E_{k}\left(\Pi_{k} \zeta-\zeta_{k}\right)\right\|_{W}=\sup _{\phi \in W^{\prime} \backslash\{0\}} \frac{\left|\phi\left(E_{k}\left(\Pi_{k} \zeta-\zeta_{k}\right)\right)\right|}{\|\phi\|_{W^{\prime}}} .
$$

Let $\phi \in W^{\prime}$ be arbitrary. We define $\xi \in Z$ and $\xi_{k} \in V_{k}$ by the following equations:

$$
\begin{array}{rlrl}
a(\xi, v) & =\phi(v) & & \forall v \in V, \\
a_{k}\left(\xi_{k}, v\right) & =\phi\left(E_{k} v\right) & \forall v \in V_{k} .
\end{array}
$$

From (R-2) we have

$$
\|\xi\|_{Z} \lesssim\|\phi\|_{W^{\prime}}
$$

Using (2.4), (3.27) and (3.28), we have

$$
\phi\left(E_{k}\left(\Pi_{k} \zeta-\zeta_{k}\right)\right)=a\left(\xi, E_{k} \Pi_{k} \zeta-\zeta\right)+a_{k}\left(\xi_{k}, \zeta-\zeta_{k}\right)+a_{k}\left(\xi-\xi_{k}, \zeta\right) .
$$

The terms on the right-hand side of (3.30) can be estimated as follows.

Using (D) and (3.4), we have

$$
\left|a\left(\xi, E_{k} \Pi_{k} \zeta-\zeta\right)\right| \lesssim\|\xi\|_{Z}\left\|E_{k} \Pi_{k} \zeta-\zeta\right\|_{W} \lesssim h_{k}^{2 \alpha}\|\xi\|_{Z}\|\zeta\|_{Z}
$$

It follows from (П-2), (N-2) and (3.23) that

$$
\begin{aligned}
\left|a_{k}\left(\xi_{k}, \zeta-\zeta_{k}\right)\right| & \lesssim\left|a_{k}\left(\xi_{k}-\Pi_{k} \xi, \zeta-\zeta_{k}\right)\right|+\left|a_{k}\left(\Pi_{k} \xi, \zeta-\zeta_{k}\right)\right| \\
& \lesssim h_{k}^{2 \alpha}\|\xi\|_{Z}\|\zeta\|_{Z} .
\end{aligned}
$$

Similarly, we have

$$
\left|a_{k}\left(\xi-\xi_{k}, \zeta\right)\right| \lesssim h_{k}^{2 \alpha}\|\xi\|_{Z}\|\zeta\|_{Z} .
$$

The estimate (3.24) now follows by combining (3.26) and (3.29)-(3.33).

The following corollary is an immediate consequence of (П-2), (ЕП), (3.1), (3.4), (3.17), (3.23) and (3.24).

Corollary 3.6. Let $\zeta \in Z$ and $\zeta_{k} \in V_{k}$ be related by (2.4). Then the following estimates hold:

$$
\left\|\zeta-E_{k} \zeta_{k}\right\|_{V} \lesssim h_{k}^{\alpha}\|\zeta\|_{Z} \quad \text { and } \quad\left\|\zeta-E_{k} \zeta_{k}\right\|_{W} \lesssim h_{k}^{2 \alpha}\|\zeta\|_{Z} .
$$


Remark. Let $\zeta \in Z$ and $\zeta_{k} \in V_{k}$ be the solutions of the continuous problem $a(\zeta, v)=$ $\phi(v) \forall v \in V$ and the discrete problem $a_{k}\left(\zeta_{k}, v\right)=\phi\left(E_{k} v\right) \forall v \in V_{k}$. Because of the presence of the operator $E_{k}$, the discrete problem is well-posed for $\phi$ in some negative order Sobolev spaces even though $V_{k}$ is nonconforming. Theorem 3.5 and Corollary 3.6 give the discretization error estimates for these new nonconforming finite element methods.

Lemma 3.7. Let $\zeta \in Z$, and let $\zeta_{k} \in V_{k}$ and $\zeta_{k-1} \in V_{k-1}$ be defined by

$$
\begin{aligned}
a_{k}\left(\zeta_{k}, v\right) & =a\left(\zeta, E_{k} v\right) & & \forall v \in V_{k}, \\
a_{k-1}\left(\zeta_{k-1}, v\right) & =a\left(\zeta, E_{k-1} v\right) & & \forall v \in V_{k-1} .
\end{aligned}
$$

Then the following estimate holds:

$$
\left\|\zeta_{k-1}-P_{k}^{k-1} \zeta_{k}\right\|_{\ell-\alpha, k-1} \lesssim h_{k}^{2 \alpha}\|\zeta\|_{Z}
$$

Proof. Again, by (3.17) and duality we have

$$
\begin{aligned}
\left\|\zeta_{k-1}-P_{k}^{k-1} \zeta_{k}\right\|_{\ell-\alpha, k-1} & \approx\left\|E_{k-1}\left(\zeta_{k-1}-P_{k}^{k-1} \zeta_{k}\right)\right\|_{W} \\
& =\sup _{\phi \in W^{\prime} \backslash\{0\}} \frac{\left|\phi\left(E_{k-1}\left(\zeta_{k-1}-P_{k}^{k-1} \zeta_{k}\right)\right)\right|}{\|\phi\|_{W^{\prime}}} .
\end{aligned}
$$

Let $\xi \in Z, \xi_{k} \in V_{k}$ and $\xi_{k-1} \in V_{k-1}$ be defined by

$$
\begin{aligned}
a(\xi, v) & =\phi(v) & & \forall v \in V, \\
a_{k}\left(\xi_{k}, v\right) & =\phi\left(E_{k} v\right) & & \forall v \in V_{k}, \\
a_{k-1}\left(\xi_{k-1}, v\right) & =\phi\left(E_{k-1} v\right) & & \forall v \in V_{k-1} .
\end{aligned}
$$

Again, the estimate (3.29) holds. Using (D), (2.8), (3.34), (3.35) and (3.40), we find that

$$
\begin{aligned}
\mid \phi( & \left.E_{k-1}\left(\zeta_{k-1}-P_{k}^{k-1} \zeta_{k}\right)\right) \mid \\
& =\left|a_{k-1}\left(\zeta_{k-1}-P_{k}^{k-1} \zeta_{k}, \xi_{k-1}\right)\right| \\
& =\left|a_{k-1}\left(\zeta_{k-1}, \xi_{k-1}\right)-a_{k}\left(\zeta_{k}, I_{k-1}^{k} \xi_{k-1}\right)\right| \\
& =\left|a\left(\zeta, E_{k-1} \xi_{k-1}-E_{k} I_{k-1}^{k} \xi_{k-1}\right)\right| \\
& \lesssim\|\zeta\|_{Z}\left\|E_{k-1} \xi_{k-1}-E_{k} I_{k-1}^{k} \xi_{k-1}\right\|_{W} .
\end{aligned}
$$

On the other hand, by (M), (3.4), (3.12), (3.14), (3.17), (3.38)-(3.40) and Theorem 3.5 , we have

$$
\begin{aligned}
& \left\|E_{k-1} \xi_{k-1}-E_{k} I_{k-1}^{k} \xi_{k-1}\right\|_{W} \\
& \lesssim \\
& \quad+\left\|E_{k-1}\left(\xi_{k-1}-\Pi_{k-1} \xi\right)\right\|_{W}+\left\|E_{k-1} \Pi_{k-1} \xi-\xi\right\|_{W} \\
& \quad+\left\|\xi-E_{k} \Pi_{k} \xi\right\|_{W}+\left\|E_{k}\left(\Pi_{k} \xi-I_{k-1}^{k} \Pi_{k-1} \xi\right)\right\|_{W} \\
& \quad+\left\|E_{k} I_{k-1}^{k}\left(\Pi_{k-1} \xi-\xi_{k-1}\right)\right\|_{W} \lesssim h_{k}^{2 \alpha}\|\xi\|_{Z} .
\end{aligned}
$$

The lemma now follows from (3.29) and (3.37)-(3.42).

Finally we derive within our abstract framework the discretization error estimates for the standard (nonconforming) discretization.

Theorem 3.8. Let $F \in X^{\prime}, u \in Z$, and $u_{k} \in V_{k}$ be such that

$$
\begin{aligned}
& a(u, v)=F(v) \forall v \in V, \\
& a_{k}\left(u_{k}, v\right)=F(v) \quad \forall v \in V_{k} .
\end{aligned}
$$


Then the following estimates hold:

$$
\begin{aligned}
\left\|u-u_{k}\right\|_{k} & \lesssim h_{k}^{\alpha}\|u\|_{Z}+h_{k}^{\ell}\|F\|_{X^{\prime}}, \\
\left\|\Pi_{k} u-u_{k}\right\|_{\ell-\alpha, k} & \lesssim h_{k}^{2 \alpha}\|u\|_{Z}+h_{k}^{\ell+\alpha}\|F\|_{X^{\prime}} .
\end{aligned}
$$

Proof. Let $u_{k}^{\prime} \in V_{k}$ satisfy

$$
a_{k}\left(u_{k}^{\prime}, v\right)=F\left(E_{k} v\right) \quad \forall v \in V_{k} .
$$

Theorem 3.5 implies that

$$
\begin{aligned}
\left\|u-u_{k}^{\prime}\right\|_{k} & \lesssim h_{k}^{\alpha}\|u\|_{Z}, \\
\left\|\Pi_{k} u-u_{k}^{\prime}\right\|_{\ell-\alpha, k} & \lesssim h_{k}^{2 \alpha}\|u\|_{Z} .
\end{aligned}
$$

From duality we have

$$
\left\|u_{k}-u_{k}^{\prime}\right\|_{k}=\sup _{v \in V_{k} \backslash\{0\}} \frac{\left|a_{k}\left(u_{k}-u_{k}^{\prime}, v\right)\right|}{\|v\|_{k}} .
$$

Using (E), (3.44) and (3.47), we obtain

$$
\left|a_{k}\left(u_{k}-u_{k}^{\prime}, v\right)\right|=\left|F\left(v-E_{k} v\right)\right| \lesssim h_{k}^{\ell}\|F\|_{X^{\prime}}\|v\|_{k} .
$$

Combining (3.50) and (3.51), we have

$$
\left\|u_{k}-u_{k}^{\prime}\right\|_{k} \lesssim h_{k}^{\ell}\|F\|_{X^{\prime}} .
$$

The estimate (3.45) follows from (3.48) and (3.52).

By (3.17) and duality, we have

$$
\left\|u_{k}^{\prime}-u_{k}\right\|_{\ell-\alpha, k} \approx\left\|E_{k}\left(u_{k}^{\prime}-u_{k}\right)\right\|_{W}=\sup _{\phi \in W^{\prime} \backslash\{0\}} \frac{\left|\phi\left(E_{k}\left(u_{k}^{\prime}-u_{k}\right)\right)\right|}{\|\phi\|_{W^{\prime}}} .
$$

Let $\xi \in Z$ and $\xi_{k} \in V_{k}$ satisfy (3.27), (3.28) and (3.29). It follows that

$$
\phi\left(E_{k}\left(u_{k}^{\prime}-u_{k}\right)\right)=a_{k}\left(\xi_{k}-\Pi_{k} \xi, u_{k}^{\prime}-u_{k}\right)+a_{k}\left(\Pi_{k} \xi, u_{k}^{\prime}-u_{k}\right) .
$$

From (П-2) and (3.52) we have

$$
\left|a_{k}\left(\xi_{k}-\Pi_{k} \xi, u_{k}^{\prime}-u_{k}\right)\right| \lesssim h_{k}^{\ell+\alpha}\|F\|_{X^{\prime}}\|\xi\|_{Z} .
$$

On the other hand, from (П-2), (ЕП), (3.44) and (3.47) we obtain

$$
\begin{aligned}
\left|a_{k}\left(\Pi_{k} \xi, u_{k}^{\prime}-u_{k}\right)\right| & =\left|F\left(E_{k} \Pi_{k} \xi-\Pi_{k} \xi\right)\right| \\
& \leq\|F\|_{X^{\prime}}\left(\left\|E_{k} \Pi_{k} \xi-\xi\right\|_{X}+\left\|\xi-\Pi_{k} \xi\right\|_{X}\right) \\
& \lesssim h_{k}^{\ell+\alpha}\|F\|_{X^{\prime}}\|\xi\|_{Z} .
\end{aligned}
$$

Combining (3.29) and (3.53)-(3.56), we have

$$
\left\|u_{k}^{\prime}-u_{k}\right\|_{\ell-\alpha, k} \lesssim h_{k}^{\ell+\alpha}\|F\|_{X^{\prime}}
$$

The estimate (3.46) follows from (3.49) and (3.57).

Remark. The estimate (3.45) is the Assumption A4 in [65].

The following corollary is an immediate consequence of (П-2), (ЕП), (3.1), (3.4), (3.17), (3.45) and (3.46). 
Corollary 3.9. Let $F, u$ and $u_{k}$ be as in Theorem 3.8. Then the following estimates hold:

$$
\begin{aligned}
\left\|u-E_{k} u_{k}\right\|_{V} & \lesssim h_{k}^{\alpha}\|u\|_{Z}+h_{k}^{\ell}\|F\|_{X^{\prime}}, \\
\left\|u-E_{k} u_{k}\right\|_{W} & \lesssim h_{k}^{2 \alpha}\|u\|_{Z}+h_{k}^{\ell+\alpha}\|F\|_{X^{\prime}} .
\end{aligned}
$$

\section{Convergence analysis}

In this section we establish the convergence results for the multigrid algorithms. First we investigate the convergence of the $W$-cycle algorithm. Following the methodology in [6], we start with the convergence analysis of the two-grid algorithm, where we assume that the residual equation is solved exactly on the coarser grid, i.e., the $q_{2}$ in the correction step is replaced by

$$
q=A_{k-1}^{-1} \bar{g} .
$$

Let $z$ be the exact solution of (1.1), and let $e_{i}=z-z_{i}$ for $i=0, \ldots, m$, where $m=m_{1}+m_{2}+1$. In order to relate the final error $e_{m}$ to the initial error $e_{0}$, we introduce the operator $R_{k}$ defined by

$$
R_{k}=I-\frac{1}{\Lambda_{k}} A_{k} .
$$

From the pre-smoothing step (1.2) and the post-smoothing step (1.4), we have

$$
e_{j}=R_{k} e_{j-1}, \quad j=1,2, \ldots, m_{1}, m_{1}+2, \ldots m .
$$

Since $\Lambda_{k}$ dominates the spectral radius of $A_{k}$, it is easy to see that

$$
\left\|R_{k} v\right\|_{s, k} \leq\|v\|_{s, k} \quad \forall v \in V_{k}, s \in \mathbb{R} .
$$

From (2.9), the correction step of the two-grid algorithm, and (4.1), we have

$$
e_{m_{1}+1}=e_{m_{1}}-I_{k-1}^{k} q=e_{m_{1}}-I_{k-1}^{k} A_{k-1}^{-1} I_{k}^{k-1} A_{k} e_{m_{1}}=\left(I-I_{k-1}^{k} P_{k}^{k-1}\right) e_{m_{1}} .
$$

It follows from (4.3) and (4.5) that

$$
e_{m}=R_{k}^{m_{2}}\left(I-I_{k-1}^{k} P_{k}^{k-1}\right) R_{k}^{m_{1}} e_{0} .
$$

Lemma 4.1. We have the following smoothing property:

$$
\begin{aligned}
& \left\|R_{k}^{n} v\right\|_{s, k} \lesssim h_{k}^{-\beta}[\max (1, n)]^{-\beta /(2 \ell)}\|v\|_{s-\beta, k} \\
& \qquad \text { for any } s \in \mathbb{R}, \beta \geq 0, n=0,1,2, \ldots .
\end{aligned}
$$

Proof. For $n \geq 1$, the proof of (4.7) is standard (cf., [6]). For $n=0$, the estimate follows from (3.10).

The following estimate on the operator $I-I_{k-1}^{k} P_{k}^{k-1}$ is the crux of the convergence analysis.

Lemma 4.2. We have the following approximation property:

$$
\left\|\left(I-I_{k-1}^{k} P_{k}^{k-1}\right) v\right\|_{\ell-\alpha, k} \lesssim h_{k}^{2 \alpha}\|v\|_{\ell+\alpha, k} \quad \forall v \in V_{k} .
$$

Proof. From (3.17) and duality we have

$$
\left\|\left(I-I_{k-1}^{k} P_{k}^{k-1}\right) v\right\|_{\ell-\alpha, k} \approx \sup _{\phi \in W^{\prime} \backslash\{0\}} \frac{\left|\phi\left(E_{k}\left(I-I_{k-1}^{k} P_{k}^{k-1}\right) v\right)\right|}{\|\phi\|_{W^{\prime}}} .
$$


Let $\phi \in W^{\prime}$ be arbitrary. We define $\zeta \in Z, \zeta_{k} \in V_{k}$ and $\zeta_{k-1} \in V_{k-1}$ by

$$
\begin{aligned}
a(\zeta, v) & =\phi(v) & & \forall v \in V, \\
a_{k}\left(\zeta_{k}, v\right) & =\phi\left(E_{k} v\right) & & \forall v \in V_{k}, \\
a_{k-1}\left(\zeta_{k-1}, v\right) & =\phi\left(E_{k-1} v\right) & & \forall v \in V_{k-1} .
\end{aligned}
$$

From (R-2), we have

$$
\|\zeta\|_{Z} \lesssim\|\phi\|_{W^{\prime}}
$$

Using (2.8) and (4.11), we find

$$
\begin{aligned}
\phi\left(E_{k}\right. & \left.\left(I-I_{k-1}^{k} P_{k}^{k-1}\right) v\right) \\
& =a_{k}\left(\zeta_{k},\left(I-I_{k-1}^{k} P_{k}^{k-1}\right) v\right) \\
& =a_{k}\left(\zeta_{k}, v\right)-a_{k-1}\left(P_{k}^{k-1} \zeta_{k}, P_{k}^{k-1} v\right) \\
& =a_{k}\left(\zeta_{k}-I_{k-1}^{k} \zeta_{k-1}, v\right)+a_{k-1}\left(\zeta_{k-1}-P_{k}^{k-1} \zeta_{k}, P_{k}^{k-1} v\right) .
\end{aligned}
$$

We can estimate the two terms on the last line of (4.14) by using (M), (3.11)(3.14), (4.10)-(4.12), Theorem 3.5 and Lemma 3.7 as follows:

$$
\begin{aligned}
& \left|a_{k}\left(\zeta_{k}-I_{k-1}^{k} \zeta_{k-1}, v\right)\right| \\
& \quad \lesssim\left\|\zeta_{k}-I_{k-1}^{k} \zeta_{k-1}\right\|_{\ell-\alpha, k}\|v\|_{\ell+\alpha, k} \\
& \quad \lesssim\left(\left\|\zeta_{k}-\Pi_{k} \zeta\right\|_{\ell-\alpha, k}+\left\|I_{k-1}^{k} \Pi_{k-1} \zeta-\Pi_{k} \zeta\right\|_{\ell-\alpha, k}\right. \\
& \left.\quad+\left\|I_{k-1}^{k}\left(\Pi_{k-1} \zeta-\zeta_{k-1}\right)\right\|_{\ell-\alpha, k}\right)\|v\|_{\ell+\alpha, k} \\
& \quad \lesssim h_{k}^{2 \alpha}\|\zeta\|_{Z}\|v\|_{\ell+\alpha, k}, \\
& \quad \lesssim\left\|\zeta_{k-1}-P_{k}^{k-1} \zeta_{k}\right\|_{\ell-\alpha, k-1}\left\|P_{k-1}^{k-1} v\right\|_{\ell+\alpha, k-1} \\
& \quad \lesssim h_{k}^{2 \alpha-1}\|\zeta\|_{Z}\|v\|_{\ell+\alpha, k} .
\end{aligned}
$$

The estimate (4.8) now follows from (4.9) and (4.13)-(4.16).

Theorem 4.3 (Convergence of the two-grid algorithm). For $m_{1}+m_{2}$ sufficiently large, the two-grid algorithm is a contraction in the $\|\cdot\|_{k}$ norm, with contraction number uniformly bounded away from 1 . For $m_{1}$ sufficiently large, the two-grid algorithm is also a contraction in the $\|\cdot\|_{\ell-\alpha, k}$ norm, and the contraction number is uniformly bounded away from 1.

Proof. Since the final error $e_{m}$ is related to the initial error $e_{0}$ by (4.6), we have by (3.9), (4.7) and (4.8) that

$$
\begin{aligned}
\left\|e_{m}\right\|_{k} & =\left\|R_{k}^{m_{2}}\left(I-I_{k-1}^{k} P_{k}^{k-1}\right) R_{k}^{m_{1}} e_{0}\right\|_{\ell, k} \\
& \lesssim h_{k}^{-\alpha}\left[\max \left(1, m_{2}\right)\right]^{-\alpha /(2 \ell)}\left\|\left(I-I_{k-1}^{k} P_{k}^{k-1}\right) R_{k}^{m_{1}} e_{0}\right\|_{\ell-\alpha, k} \\
& \lesssim h_{k}^{\alpha}\left[\max \left(1, m_{2}\right)\right]^{-\alpha /(2 \ell)}\left\|R_{k}^{m_{1}} e_{0}\right\|_{\ell+\alpha, k} \\
& \lesssim\left[\max \left(1, m_{2}\right)\right]^{-\alpha /(2 \ell)}\left[\max \left(1, m_{1}\right)\right]^{-\alpha /(2 \ell)}\left\|e_{0}\right\|_{k} .
\end{aligned}
$$


Similarly, we have by (4.4), (4.7) and (4.8) that

$$
\begin{aligned}
\left\|e_{m}\right\|_{\ell-\alpha, k} & =\left\|R_{k}^{m_{2}}\left(I-I_{k-1}^{k} P_{k}^{k-1}\right) R_{k}^{m_{1}} e_{0}\right\|_{\ell-\alpha, k} \\
& \leq\left\|\left(I-I_{k-1}^{k} P_{k}^{k-1}\right) R_{k}^{m_{1}} e_{0}\right\|_{\ell-\alpha, k} \\
& \lesssim h_{k}^{2 \alpha}\left\|R_{k}^{m_{1}} e_{0}\right\|_{\ell+\alpha, k} \\
& \lesssim\left[\max \left(1, m_{1}\right)\right]^{-\alpha / \ell}\left\|e_{m}\right\|_{\ell-\alpha, k} .
\end{aligned}
$$

The theorem follows from (4.17) and (4.18).

Remark. The estimate in (4.18) seems to indicate that a one-sided algorithm with only pre-smoothing may be more efficient for convergence in the $\|\cdot\|_{\ell-\alpha, k}$ norm.

The next theorem follows from (3.12), Theorem 4.3 and a standard perturbation argument (cf., [6]).

Theorem 4.4 (Convergence of the $W$-cycle multigrid algorithm). For $m_{1}+m_{2}$ sufficiently large, the $W$-cycle multigrid algorithm is a contraction in the $\|\cdot\|_{k}$ norm, with contraction number uniformly bounded away from 1 . For $m_{1}$ sufficiently large, the $W$-cycle algorithm is also a contraction in the $\|\cdot\|_{\ell-\alpha, k}$ norm, and the contraction number is uniformly bounded away from 1.

Let $F \in X^{\prime}, u \in Z$ and $u_{k} \in V_{k}$ be such that (3.43) and (3.44) hold. We can find an approximate solution for (3.44) by the following full multigrid method.

The full multigrid $W$-cycle algorithm. For $k=1$, the approximate solution $\hat{u}_{1} \in V_{1}$ is obtained by a direct method.

For $k>1$, the approximate solution $\hat{u}_{k} \in V_{k}$ is obtained recursively from

$$
\begin{aligned}
u_{k, 0} & =I_{k-1}^{k} \hat{u}_{k-1} \\
u_{k, j} & =W M G\left(k, u_{k, j-1}, f_{k}\right), \quad 1 \leq j \leq r, \\
\hat{u}_{k} & =u_{k, r},
\end{aligned}
$$

where $r$ is a positive integer independent of $k$ and $f_{k} \in V_{k}$ is defined by

$$
\left(f_{k}, v\right)_{k}=F(v) \quad \forall v \in V_{k} .
$$

Theorem 4.5. Let $F \in X^{\prime}, u \in Z$ and $u_{k} \in V_{k}$ be such that (3.43) and (3.44) hold. Let $m_{1}+m_{2}$ (resp., $m_{1}$ ) be sufficiently large so that the $W$-cycle algorithms are contractions in the $\|\cdot\|_{k}$ (resp., $\|\cdot\|_{\ell-\alpha, k}$ ) norms with contraction numbers uniformly bounded away from 1. Then, for $r$ sufficiently large, the following estimates hold for the approximate solutions $\hat{u}_{k}(k=1,2, \ldots)$ obtained by the full multigrid algorithm:

$$
\begin{aligned}
\left\|u_{k}-\hat{u}_{k}\right\|_{k} & \lesssim h_{k}^{\alpha}\|u\|_{Z}+h_{k}^{\ell}\|F\|_{X^{\prime}} \\
\left\|u_{k}-\hat{u}_{k}\right\|_{\ell-\alpha, k} & \lesssim h_{k}^{2 \alpha}\|u\|_{Z}+h_{k}^{\ell+\alpha}\|F\|_{X^{\prime}} .
\end{aligned}
$$


Proof. By (M), (П-2), (3.3), (3.9), (3.12), (3.14), (3.16), (3.45) and Theorem 3.8 we have

$$
\begin{gathered}
\left\|u_{k}-I_{k-1}^{k} u_{k-1}\right\|_{k} \leq\left\|u_{k}-\Pi_{k} u\right\|_{k}+\left\|\Pi_{k} u-I_{k-1}^{k} \Pi_{k-1} u\right\|_{k} \\
+\left\|I_{k-1}^{k}\left(\Pi_{k-1} u-u_{k-1}\right)\right\|_{k} \\
\lesssim h_{k}^{\alpha}\|u\|_{Z}+h_{k}^{\ell}\|F\|_{X^{\prime}} \\
\left\|u_{k}-I_{k-1}^{k} u_{k-1}\right\|\left\|_{\ell-\alpha, k} \leq\right\| u_{k}-\Pi_{k} u\left\|_{\ell-\alpha, k}+\right\| \Pi_{k} u-I_{k-1}^{k} \Pi_{k-1} u \|_{\ell-\alpha, k} \\
\quad+\left\|I_{k-1}^{k}\left(\Pi_{k-1} u-u_{k-1}\right)\right\|_{\ell-\alpha, k} \\
\lesssim h_{k}^{2 \alpha}\|u\|_{Z}+h_{k}^{\ell+\alpha}\|F\|_{X^{\prime}}
\end{gathered}
$$

Let $k>1$. By (4.19) and the assumption on the $W$-cycle algorithm, there exists a positive $\delta$ such that $\delta<1$ and

$$
\begin{aligned}
\left\|u_{k}-\hat{u}_{k}\right\|_{k} & \leq \delta^{r}\left\|u_{k}-I_{k-1}^{k} \hat{u}_{k-1}\right\|_{k} & & \text { for } k=1,2, \ldots, \\
\left\|u_{k}-\hat{u}_{k}\right\|_{\ell-\alpha, k} & \leq \delta^{r}\left\|u_{k}-I_{k-1}^{k} \hat{u}_{k-1}\right\|_{\ell-\alpha, k} & & \text { for } k=1,2, \ldots .
\end{aligned}
$$

Combining (3.3), (4.22) and (4.24), we obtain

$$
\begin{aligned}
\left\|u_{k}-\hat{u}_{k}\right\|_{k} & \leq \delta^{r}\left[\left\|u_{k}-I_{k-1}^{k} u_{k-1}\right\|_{k}+\left\|I_{k-1}^{k}\left(u_{k-1}-\hat{u}_{k-1}\right)\right\|_{k}\right] \\
& \leq \delta^{r} C^{\prime}\left[\left(h_{k}^{\alpha}\|u\|_{Z}+h_{k}^{\ell}\|F\|_{X^{\prime}}\right)+\left\|u_{k-1}-\hat{u}_{k-1}\right\|_{k}\right],
\end{aligned}
$$

where $C^{\prime}$ is independent of $k$.

Similarly, using (3.12), (4.23) and (4.25), we obtain

$$
\left\|u_{k}-\hat{u}_{k}\right\|_{\ell-\alpha, k} \leq \delta^{r} C^{\prime \prime}\left[\left(h_{k}^{2 \alpha}\|u\|_{Z}+h_{k}^{\ell+\alpha}\|F\|_{X^{\prime}}\right)+\left\|u_{k-1}-\hat{u}_{k-1}\right\|_{\ell-\alpha, k}\right],
$$

where $C^{\prime \prime}$ is independent of $k$.

Since $0<\alpha \leq \ell$, it follows from (M) and iterations of (4.26) and (4.27) that

$$
\begin{aligned}
\left\|u_{k}-\hat{u}_{k}\right\|_{k} & \leq\left[\sum_{j=1}^{k}\left(\frac{C^{\prime} \delta^{r}}{C_{1}^{\ell}}\right)^{j}\right]\left(h_{k}^{\alpha}\|u\|_{Z}+h_{k}^{\ell}\|F\|_{X^{\prime}}\right), \\
\left\|u_{k}-\hat{u}_{k}\right\|_{\ell-\alpha, k} & \leq\left[\sum_{j=1}^{k}\left(\frac{C^{\prime \prime} \delta^{r}}{C_{1}^{\ell+\alpha}}\right)^{j}\right]\left(h_{k}^{2 \alpha}\|u\|_{Z}+h_{k}^{\ell+\alpha}\|F\|_{X^{\prime}}\right) .
\end{aligned}
$$

The estimates (4.20) and (4.21) follow from (4.28) and (4.29) for $r$ sufficiently large.

The following corollary is an immediate consequence of (3.1), (3.17), Theorem 3.8, Corollary 3.9 and Theorem 4.5.

Corollary 4.6. The following estimates hold under the assumptions of Theorem 4.5:

$$
\begin{gathered}
\left\|u-\hat{u}_{k}\right\|_{k}+\left\|u-E_{k} \hat{u}_{k}\right\|_{V} \lesssim h_{k}^{\alpha}\|u\|_{Z}+h_{k}^{\ell}\|F\|_{X^{\prime}}, \\
\left\|\Pi_{k} u-\hat{u}_{k}\right\|_{\ell-\alpha, k}+\left\|u-E_{k} \hat{u}_{k}\right\|_{W} \lesssim h_{k}^{2 \alpha}\|u\|_{Z}+h_{k}^{\ell+\alpha}\|F\|_{X^{\prime}} .
\end{gathered}
$$

Remark. For fourth order problems, pointwise convergence of $\hat{u}_{k}$ follows from (4.31) and the Sobolev inequality (cf., the remark after Example 6.1). 
Next we consider the symmetric variable $V$-cycle algorithm as a preconditioner. Let $B_{k}: V_{k} \longrightarrow V_{k}$ be defined by

$$
B_{k} g=\mathcal{V} M G(k, 0, g) .
$$

It can be shown by mathematical induction that $B_{k}$ is a linear symmetric positive definite operator with respect to $(\cdot, \cdot)_{k}$ (cf., Theorem 4.5 in [10]). Therefore $B_{k} A_{k}$ is symmetric positive definite with respect to $a_{k}(\cdot, \cdot)$. Our goal is to estimate the condition number of $B_{k} A_{k}$ with respect to the energy norm $\|\cdot\|_{k}$ induced by $a_{k}(\cdot, \cdot)$.

The following lemma furnishes the crucial "regularity and approximation" estimate in the Bramble-Pasciak-Xu theory for the symmetric variable $V$-cycle multigrid preconditioner.

Lemma 4.7. The following estimate holds:

$$
\left|a_{k}\left(\left(I-I_{k-1}^{k} P_{k}^{k-1}\right) v, v\right)\right| \lesssim\left(\frac{\left(A_{k} v, A_{k} v\right)_{k}}{\lambda_{k}}\right)^{\alpha / \ell}\left(a_{k}(v, v)\right)^{1-(\alpha / \ell)},
$$

for all $v \in V_{k}$, where $\lambda_{k}\left(=\rho\left(A_{k}\right)\right)$ is the largest eigenvalue of $A_{k}$.

Proof. Let $v \in V_{k}$ be arbitrary. Using (3.7), (3.11) and (4.8), we have

$$
\begin{aligned}
\left|a_{k}\left(\left(I-I_{k-1}^{k} P_{k}^{k-1}\right) v, v\right)\right| & \lesssim\left\|\left(I-I_{k-1}^{k} P_{k}^{k-1}\right) v\right\|_{\ell-\alpha, k}\|v\|_{\ell+\alpha, k} \\
& \lesssim h_{k}^{2 \alpha}\|v\|_{\ell+\alpha, k}^{2}=h_{k}^{2 \alpha}\left(A_{k}^{1+(\alpha / \ell)} v, v\right)_{k} .
\end{aligned}
$$

Hölder's inequality implies that

$$
\left(A_{k}^{1+(\alpha / \ell)} v, v\right)_{k} \leq\left(A_{k} v, A_{k} v\right)_{k}^{\alpha / \ell}\left(A_{k} v, v\right)_{k}^{1-(\alpha / \ell)} .
$$

The estimate (4.32) follows from (4.33), (4.34) and (2.6).

We can now simply apply the Bramble-Pasciak-Xu theory ([10], [12], [13]) to obtain the following theorem.

Theorem 4.8. The condition number of $B_{k} A_{k}$ with respect to the energy norm $\|\cdot\|_{k}$ is bounded by a positive constant which is independent of the mesh parameter $k$.

\section{Applications to a model Second order problem}

In this section we apply our theory to the Poisson equation with homogeneous Dirichlet boundary condition. Let $\Omega$ be a polygonal domain in $\mathbb{R}^{2}$ and $f \in L^{2}(\Omega)$. Consider the following boundary value problem:

$$
-\Delta u=f \quad \text { in } \Omega \text { and } \quad u=0 \quad \text { on } \partial \Omega .
$$

Let $V=H_{0}^{1}(\Omega)$, and let $a(\cdot, \cdot)$ on $V \times V$ be defined by $a\left(v_{1}, v_{2}\right)=\int_{\Omega} \nabla v_{1} \cdot \nabla v_{2} d x$. Conditions (B) and (C) follow from the Cauchy-Schwarz inequality and the Poincaré inequality (cf., [48]), respectively.

The weak formulation of (5.1) is to find $u \in V$ such that

$$
a(u, v)=\int_{\Omega} f v d x \quad \forall v \in V .
$$


By the elliptic regularity theory for non-smooth domains (cf., [36], [39], [40], [41]), there exists $\alpha \in\left(\frac{1}{2}, 1\right]$ such that for $f \in H^{-1+\alpha}(\Omega)$, the solution $u$ of (5.2) belongs to $H^{1+\alpha}(\Omega)$ and

$$
\|u\|_{H^{1+\alpha}(\Omega)} \lesssim\|f\|_{H^{-1+\alpha}(\Omega)} .
$$

Let $Z=H^{1+\alpha}(\Omega) \cap H_{0}^{1}(\Omega)$ and $W=H_{0}^{1-\alpha}(\Omega)\left(=H^{1-\alpha}(\Omega)\right.$ since $\left.1-\alpha<1 / 2\right)$. Clearly, (R-1) holds and (R-2) follows from (5.3).

Let $X=L^{2}(\Omega)$. From interpolation of Sobolev spaces (cf., [61] and [57]) we have

$$
Z=\left[H_{0}^{1}(\Omega), H^{2}(\Omega) \cap H_{0}^{1}(\Omega)\right]_{\alpha},
$$

and $W=\left[L^{2}(\Omega), H_{0}^{1}(\Omega)\right]_{1-\alpha}$. In particular, the condition (C-3) holds.

The Laplacian $\Delta$ is a bounded linear operator from $H^{2}(\Omega)$ to $L^{2}(\Omega)$, and from $H^{1}(\Omega)$ to $H^{-1}(\Omega)$. Therefore by interpolation (cf., [61]) we have

$$
\|\Delta \zeta\|_{H^{-1+\alpha}(\Omega)} \lesssim\|\zeta\|_{H^{1+\alpha}(\Omega)} \quad \forall \zeta \in H^{1+\alpha}(\Omega) .
$$

Let $\zeta \in Z$ and $v \in V$. There exists a sequence $\phi_{n} \in C_{0}^{\infty}(\Omega)$ (the space of $C^{\infty}$ functions with compact supports in $\Omega$ ) which converges to $v \in V$. Since $H_{0}^{1}(\Omega) \hookrightarrow H_{0}^{1-\alpha}(\Omega)$, the sequence $\phi_{n}$ also converges to $v \in H_{0}^{1-\alpha}(\Omega)$. Therefore, we have

$$
a(\zeta, v)=\lim _{n \rightarrow \infty} \int_{\Omega} \nabla \zeta \cdot \nabla \phi_{n} d x=\lim _{n \rightarrow \infty}\left(-\Delta \zeta, \phi_{n}\right)=(-\Delta \zeta, v),
$$

where $(\cdot, \cdot)$ denotes the canonical duality bilinear form between $H^{-1+\alpha}(\Omega)$ and $H_{0}^{1-\alpha}(\Omega)$. The duality estimate (D) now follows from (5.5) and (5.6).

We now consider finite element multigrid methods for (5.2).

Example 5.1. Let $\left\{\mathcal{T}_{k}\right\}$ be a sequence of quasi-uniform triangulations (cf., [30], [27]) of $\Omega$. For simplicity we may assume that $\mathcal{T}_{k+1}$ is obtained by connecting the midpoints of the edges of the triangles in $\mathcal{T}_{k}$. Therefore, $(\mathrm{M})$ holds for $C_{1}=C_{2}=$ $1 / 2$.

Let $V_{k}^{*}=\left\{v \in L^{2}(\Omega):\left.v\right|_{T}\right.$ is linear for all $T \in \mathcal{T}_{k}, v$ is continuous at the midpoints of interelement boundaries $\}$ be the $\mathcal{P}_{1}$ nonconforming finite element space associated with $\mathcal{T}_{k}$ (cf., [35]), and let $\tilde{V}_{k}^{*}=\left\{v \in H^{1}(\Omega):\left.v\right|_{T}\right.$ is quadratic for all $\left.T \in \mathcal{T}_{k}\right\}$ be the $\mathcal{P}_{2}$ conforming finite element space associated with $\mathcal{T}_{k}$. The space $V_{k}$ (resp., $\tilde{V}_{k}$ ) is the subspace of $V_{k}^{*}$ (resp., $\tilde{V}_{k}^{*}$ ) whose members vanish at the boundary nodes. Note that $V_{k} \preceq \tilde{V}_{k}$ and the conditions (C-1) and (C-2) clearly hold. The finite element space $V_{k}^{*}$ is equipped with the inner product $(\cdot, \cdot)_{k}$ defined by $\left(v_{1}, v_{2}\right)_{k}=h_{k}^{2} \sum_{m} v_{1}(m) v_{2}(m)$, where the summation is taken over all the midpoints in the triangulation $\mathcal{T}_{k}$. The equivalence of $(v, v)_{k}$ and $(v, v)_{L^{2}(\Omega)}$ is standard. Hence $(\mathrm{P})$ holds.

Let $a_{k}(\cdot, \cdot)$ be defined by $a_{k}\left(v_{1}, v_{2}\right)=\sum_{T \in \mathcal{T}_{k}} \int_{T} \nabla v_{1} \cdot \nabla v_{2} d x$. Then (I) is a standard inverse estimate (cf., [30], [27]). The discrete problem for (5.2) is to find $u_{k} \in V_{k}$ such that

$$
a_{k}\left(u_{k}, v\right)=\int_{\Omega} f v d x \quad \forall v \in V_{k} .
$$

The interpolation operator $\Pi_{k}: V \longrightarrow V_{k}$ is defined by

$$
\left(\Pi_{k} v\right)(m)=\frac{1}{|e|} \int_{e} v d s,
$$


where $m$ is the midpoint of the edge $e$. Note that

$$
\left.\left(\Pi_{k} \zeta\right)\right|_{T}=\left.\zeta\right|_{T} \text { if }\left.\zeta\right|_{T} \text { is linear. }
$$

The following estimate can be found in [35]:

$$
\left\|\zeta-\Pi_{k} \zeta\right\|_{L^{2}(T)}+h_{k}\left|\zeta-\Pi_{k} \zeta\right|_{H^{1}(T)} \lesssim h_{k}^{\beta}|\zeta|_{H^{\beta}(T)}, \quad \beta=1,2,
$$

for all $T \in \mathcal{T}_{k}$ and $\zeta \in H^{\beta}(\Omega) \cap H_{0}^{1}(\Omega)$. The estimate (П-1) follows from (5.10) with $\beta=1$, and the estimate (П-2) follows from (П-1), (5.4), (5.10) with $\beta=2$ and interpolation.

The operators $E_{k}: V_{k} \longrightarrow \tilde{V}_{k}$ and $F_{k}: \tilde{V}_{k} \longrightarrow V_{k}$ are defined by

$$
\begin{cases}\left(E_{k} v\right)(m)=v(m) & \text { for all internal midpoints } m \in \mathcal{T}_{k}, \\ \left(E_{k} v\right)(p)=\text { average of } v_{i}(p) & \text { for all internal vertices } p \in \mathcal{T}_{k}\end{cases}
$$

where $v_{i}=\left.v\right|_{T_{i}}$ and $T_{i} \in \mathcal{T}_{k}$ contains $p$ as a vertex, and

$$
\left(F_{k} \tilde{v}\right)(m)=\tilde{v}(m) \quad \text { for all midpoints } m \in \mathcal{T}_{k} .
$$

Note that $F_{k}$ is well-defined because $V_{k} \preceq \tilde{V}_{k}$.

The relation (FE) is trivial, and the estimates $(\mathrm{E})$ and $(\mathrm{F})$ can be found in [24]. For the proof of (Eח), it is convenient (because we can ignore the boundary conditions) to introduce the operator $E_{k}^{*}: V_{k}^{*} \longrightarrow \tilde{V}_{k}^{*}$ which is defined by the same formula in (5.11) for all midpoints and vertices of $\mathcal{T}_{k}$. Note that for $v \in V_{k}$, we have $E_{k}^{*} v=E_{k} v$ except at the vertices on $\partial \Omega$.

Let $T \in \mathcal{T}_{k}$, and let $S_{T}$ be the interior of the union of the closures of all the triangles in $\mathcal{T}_{k}$ neighboring $T$. We have the following estimate for $E_{k}^{*}$ (cf., [24]).

$$
\left\|E_{k}^{*} v-v\right\|_{L^{2}(T)}^{2} \lesssim h_{T}^{2} \sum_{K \in S_{T}}|v|_{H^{1}(K)}^{2} \quad \forall v \in V_{k}^{*} .
$$

It follows from (5.13) and a standard inverse estimate that

$$
\left\|E^{*} v\right\|_{L^{2}(T)} \lesssim\|v\|_{L^{2}\left(S_{T}\right)} \quad \forall v \in V_{k}^{*} .
$$

The definition of $E_{k}^{*}$ also implies that

$$
\left.\left(E_{k}^{*} \eta\right)\right|_{T}=\eta \quad \text { if }\left.\eta\right|_{S_{T}} \text { is linear. }
$$

Let $T \in \mathcal{T}_{k}$. Let $\phi$ be an arbitrary linear function on $S_{T}$ and $\tilde{\phi} \in H^{2}(\Omega)$ be an extension of $\phi$. For any $\zeta \in H^{2}(\Omega) \cap H_{0}^{1}(\Omega)$, it follows from (5.9), (5.10), (5.14) and (5.15) that

$$
\left\|E_{k}^{*} \Pi_{k} \zeta-\zeta\right\|_{L^{2}(T)} \leq\left\|E_{k}^{*} \Pi_{k}(\zeta-\tilde{\phi})\right\|_{L^{2}(T)}+\|\zeta-\tilde{\phi}\|_{L^{2}(T)} \lesssim\|\zeta-\phi\|_{L^{2}\left(S_{T}\right)} .
$$

Since $\phi$ is an arbitrary linear function on $S_{T}$, it follows from (5.16) and the BrambleHilbert lemma (cf., [11]) that

$$
\left\|E_{k}^{*} \Pi_{k} \zeta-\zeta\right\|_{L^{2}(T)} \lesssim h_{T}^{2}|\zeta|_{H^{2}\left(S_{T}\right)} .
$$

Summing (5.17) over all the triangles $T \in \mathcal{T}_{k}$, we have

$$
\left\|E_{k}^{*} \Pi_{k} \zeta-\zeta\right\|_{L^{2}(\Omega)} \lesssim h_{k}^{2}|\zeta|_{H^{2}(\Omega)} \quad \forall \zeta \in H^{2}(\Omega) \cap H_{0}^{1}(\Omega) .
$$


Since $E_{k} \Pi_{k} \zeta$ and $E_{k}^{*} \Pi_{k} \zeta$ differ only at the vertices along $\partial \Omega$, we have

$$
\begin{aligned}
\left\|E_{k} \Pi_{k} \zeta-E_{k}^{*} \Pi_{k} \zeta\right\|_{L^{2}(\Omega)}^{2} & \lesssim h_{k}^{2} \sum_{p \in \partial \Omega} \sum_{\substack{T \in \mathcal{T}_{k} \\
\bar{T} \ni p}}\left[\left.\left(\Pi_{k} \zeta\right)\right|_{T}(p)\right]^{2} \\
& \lesssim h_{k}^{2} \sum_{p \in \partial \Omega} \sum_{\substack{T \in \mathcal{T}_{k} \\
\bar{T} \ni p}}\left[\left.\left(\Pi_{k} \zeta\right)\right|_{T}(p)-\zeta(p)\right]^{2} \\
& \lesssim h_{k}^{4}|\zeta|_{H^{2}(\Omega)}^{2}
\end{aligned}
$$

by (5.10). It follows from (5.18) and (5.19) that

$$
\left\|E_{k} \Pi_{k} \zeta-\zeta\right\|_{L^{2}(\Omega)} \lesssim h_{k}^{2}|\zeta|_{H^{2}(\Omega)} \quad \forall \zeta \in H^{2}(\Omega) \cap H_{0}^{1}(\Omega) .
$$

By (5.10), (5.20) and standard inverse estimates, we have

$$
\begin{aligned}
\left\|E_{k} \Pi_{k} \zeta-\zeta\right\|_{H^{1}(\Omega)} & \lesssim h_{k}^{-1}\left\|E_{k} \Pi_{k} \zeta-\Pi_{k} \zeta\right\|_{L^{2}(\Omega)}+\left\|\Pi_{k} \zeta-\zeta\right\|_{k} \\
& \lesssim h_{k}|\zeta|_{H^{2}(\Omega)} \quad \forall \zeta \in H^{2}(\Omega) \cap H_{0}^{1}(\Omega) .
\end{aligned}
$$

On the other hand, using (E), (I) and (П-1), we have

$$
\begin{aligned}
& \left\|E_{k} \Pi_{k} \zeta-\zeta\right\|_{L^{2}(\Omega)}+h_{k}\left\|E_{k} \Pi_{k} \zeta-\zeta\right\|_{H^{1}(\Omega)} \\
& \lesssim\left\|E_{k} \Pi_{k} \zeta-\Pi_{k} \zeta\right\|_{L^{2}(\Omega)}+\left\|\Pi_{k} \zeta-\zeta\right\|_{L^{2}(\Omega)} \\
& \quad+h_{k}\left(\left\|E_{k} \Pi_{k} \zeta-\Pi_{k} \zeta\right\|_{k}+\left\|\Pi_{k} \zeta-\zeta\right\|_{k}\right) \\
& \lesssim h_{k}\|\zeta\|_{H^{1}(\Omega)} \quad \forall \zeta \in H_{0}^{1}(\Omega) .
\end{aligned}
$$

The estimate (EП) now follows from (5.4), (5.20), (5.21), (5.22) and interpolation.

Next we verify the assumptions (N-1) and (N-2). Let $\zeta \in H^{2}(\Omega) \cap H_{0}^{1}(\Omega)$, and let $\zeta_{k} \in V_{k}$ be related to $\zeta$ through (2.4). Let $v \in V_{k}+V$, then Green's formula implies that

$$
a_{k}(\zeta, v)=-\sum_{T \in \mathcal{T}_{k}} \int_{T}(\Delta \zeta) v d x+\sum_{e} \int_{e} \frac{\partial \zeta}{\partial n}[v] d s
$$

where $[v]$ denotes the jump of $v$ (in the direction of $n$ ) across the edge $e$, and the second summation is taken over all the edges of $\mathcal{T}_{k}$.

Since $E_{k} v \in H_{0}^{1}(\Omega)$, it follows from (2.4) and (5.23) that

$$
a_{k}\left(\zeta_{k}, v\right)=-\sum_{T \in \mathcal{T}_{k}} \int_{T}(\Delta \zeta) E_{k} v d x \quad \forall v \in V_{k}
$$

By subtracting (5.24) from (5.23), we obtain

$$
a_{k}\left(\zeta-\zeta_{k}, v\right)=-\sum_{T \in \mathcal{T}_{k}} \int_{T}(\Delta \zeta)\left(v-E_{k} v\right) d x+\sum_{e} \int_{e} \frac{\partial \zeta}{\partial n}[v] d s \quad \forall v \in V_{k} .
$$

Using the Cauchy-Schwarz inequality and (E), we have

$$
\left|\sum_{T \in \mathcal{T}_{k}} \int_{T}(\Delta \zeta)\left(v-E_{k} v\right) d x\right| \lesssim h_{k}\|\zeta\|_{H^{2}(\Omega)}\|v\|_{k} \quad \forall v \in V_{k} .
$$


Since $v$ is continuous at the midpoints, a standard argument (cf., [35]) shows that

$$
\left|\sum_{e} \int_{e} \frac{\partial \zeta}{\partial n}[v] d s\right| \lesssim h_{k}\|\zeta\|_{H^{2}(\Omega)}\|v\|_{k} \quad \forall v \in V_{k} .
$$

Combining (5.25)-(5.27), we have

$$
\left|a_{k}\left(\zeta-\zeta_{k}, v\right)\right| \lesssim h_{k}\|\zeta\|_{H^{2}(\Omega)}\|v\|_{k} \quad \forall v \in V_{k} .
$$

Assume now that $\zeta \in H_{0}^{1}(\Omega)$. By (2.4) and (3.1) we have

$$
a_{k}\left(\zeta_{k}, \zeta_{k}\right)=a\left(\zeta, E_{k} \zeta_{k}\right) \leq\|\zeta\|_{H^{1}(\Omega)}\left\|E_{k} \zeta_{k}\right\|_{H^{1}(\Omega)} \lesssim\|\zeta\|_{H^{1}(\Omega)}\left\|\zeta_{k}\right\|_{k} .
$$

It follows from (5.29) that

$$
\left\|\zeta_{k}\right\|_{k} \lesssim\|\zeta\|_{H^{1}(\Omega)}
$$

which then implies

$$
\left|a_{k}\left(\zeta-\zeta_{k}, v\right)\right| \leq\left\|\zeta-\zeta_{k}\right\|_{k}\|v\|_{k} \lesssim\|\zeta\|_{H^{1}(\Omega)}\|v\|_{k} \quad \forall v \in V_{k}
$$

The estimate (N-1) follows from (5.4), (5.28), (5.31) and interpolation.

From (5.25) we obtain

$$
a_{k}\left(\zeta-\zeta_{k}, \Pi_{k} \xi\right)=-\sum_{T \in \mathcal{T}_{k}} \int_{T}(\Delta \zeta)\left(\Pi_{k} \xi-E_{k} \Pi_{k} \xi\right) d x+\sum_{e} \int_{e} \frac{\partial \zeta}{\partial n}\left[\Pi_{k} \xi\right] d s
$$

for $\zeta, \xi \in H^{2}(\Omega) \cap H_{0}^{1}(\Omega)$.

It follows from the Cauchy-Schwarz inequality, (5.10) and (5.20) that

$$
\left|\sum_{T \in \mathcal{T}_{k}} \int_{T}(\Delta \zeta)\left(\Pi_{k} \xi-E_{k} \Pi_{k} \xi\right) d x\right| \lesssim h_{k}^{2}\|\zeta\|_{H^{2}(\Omega)}\|\xi\|_{H^{2}(\Omega)} .
$$

Since $\Pi_{k} \xi$ is continuous at the midpoints, we have by a standard argument (cf., [35])

$$
\left|\sum_{e} \int_{e} \frac{\partial \zeta}{\partial n}\left[\Pi_{k} \xi\right] d s\right|=\left|\sum_{e} \int_{e} \frac{\partial \zeta}{\partial n}\left[\Pi_{k} \xi-\xi\right] d s\right| \lesssim h_{k}^{2}\|\zeta\|_{H^{2}(\Omega)}\|\xi\|_{H^{2}(\Omega)} .
$$

Combining (5.32)-(5.34), we obtain

$$
\left|a_{k}\left(\zeta-\zeta_{k}, \Pi_{k} \xi\right)\right| \lesssim h_{k}^{2}\|\zeta\|_{H^{2}(\Omega)}\|\xi\|_{H^{2}(\Omega)} \quad \forall \xi, \zeta \in H^{2}(\Omega) \cap H_{0}^{1}(\Omega) .
$$

On the other hand, for $\zeta, \xi \in H_{0}^{1}(\Omega)$, we get the following trivial estimate by using (5.10) and (5.30):

$$
\left|a_{k}\left(\zeta-\zeta_{k}, \Pi_{k} \xi\right)\right| \leq\left\|\zeta-\zeta_{k}\right\|_{k}\left\|\Pi_{k} \xi\right\|_{k} \lesssim\|\zeta\|_{H^{1}(\Omega)}\|\xi\|_{H^{1}(\Omega)} .
$$

The estimate (N-2) follows from (5.4), (5.35), (5.36) and (bilinear) interpolation (cf., [7]).

Finally, we define the intergrid transfer operator $I_{k-1}^{k}$. Let $m$ be a midpoint of an edge of a triangle in $\mathcal{T}_{k}$. If $m \in \partial \Omega$, then $\left(I_{k-1}^{k} v\right)(m)=0$. If $m$ lies in the interior of a triangle in $\mathcal{T}_{k-1}$, then $\left(I_{k-1}^{k} v\right)(m)=v(m)$. Otherwise if $m$ lies on the common edge of two adjacent triangles $T_{1}$ and $T_{2}$ in $\mathcal{T}_{k-1}$, then $\left(I_{k-1}^{k} v\right)(m)=$ $\frac{1}{2}\left[\left.v\right|_{T_{1}}(m)+\left.v\right|_{T_{2}}(m)\right]$. The proof of the estimate (I-1) can be found in [14] and [18].

Let $\zeta \in H^{2}(\Omega) \cap H_{0}^{1}(\Omega)$. A slight modification of the arguments in [14] and [18] (where the nodal interpolation operator was used) gives

$$
\left\|I_{k-1}^{k} \Pi_{k-1} \zeta-\Pi_{k} \zeta\right\|_{L^{2}(\Omega)} \lesssim h_{k}^{2}\|\zeta\|_{H^{2}(\Omega)} .
$$


For $\zeta \in H_{0}^{1}(\Omega)$, using $(\mathrm{M}),(\Pi-1)$ and (I-1) we have the estimate

$$
\begin{aligned}
\| I_{k-1}^{k} & \Pi_{k-1} \zeta-\Pi_{k} \zeta \|_{L^{2}(\Omega)} \\
\lesssim & \left\|I_{k-1}^{k} \Pi_{k-1} \zeta-\Pi_{k-1} \zeta\right\|_{L^{2}(\Omega)} \\
& \quad+\left\|\Pi_{k-1} \zeta-\zeta\right\|_{L^{2}(\Omega)}+\left\|\zeta-\Pi_{k} \zeta\right\|_{L^{2}(\Omega)} \\
\lesssim & h_{k}\|\zeta\|_{H^{1}(\Omega)} .
\end{aligned}
$$

The estimate (I-2) now follows from (5.4), (5.37), (5.38) and interpolation.

We have verified all of the assumptions in Section 2 for this example. Therefore the results in Section 4 are applicable to the multigrid algorithms for (5.7).

In the next example, we omit the technical details since they can be carried out along the same lines as in Example 5.1.

Example 5.2. In this example, we assume that the sides of the polygonal domain $\Omega$ are parallel to the coordinate axes. Let $\left\{\mathcal{T}_{k}\right\}$ be a sequence of quasi-uniform "triangulations" of $\Omega$ consisting of rectangles. For simplicity we may assume that $\mathcal{T}_{k+1}$ is obtained by connecting midpoints of the opposite sides of the rectangles in $\mathcal{T}_{k}$.

Let $V_{k}=\left\{v:\left.v\right|_{\mathrm{R}} \in\left\langle 1, x_{1}, x_{2}, x_{1}^{2}-x_{2}^{2}\right\rangle \forall \mathrm{R} \in \mathcal{T}_{k}, v\right.$ is continuous at the midpoints of the interelement boundaries and vanishes at the midpoints on $\partial \Omega\}$ be the nonconforming "rotated" bilinear element (cf., [52]), and $\tilde{V}_{k}=\left\{v \in H_{0}^{1}(\Omega):\left.v\right|_{\mathrm{R}}\right.$ is biquadratic for all $\left.\mathrm{R} \in \mathcal{T}_{k}\right\}$ be the conforming $\mathcal{Q}_{2}$ finite element space. Note that $V_{k} \preceq \tilde{V}_{k}$. The inner product for $V_{k}$ is defined by $\left(v_{1}, v_{2}\right)_{k}=h_{k}^{2} \sum_{m} v_{1}(m) v_{2}(m)$, where the summation is taken over all internal midpoints $m$ of the triangulation $\mathcal{T}_{k}$.

Let $a_{k}(\cdot, \cdot)$ be defined by $a_{k}\left(v_{1}, v_{2}\right)=\sum_{\mathrm{R} \in \mathcal{T}_{k}} \int_{\mathrm{R}} \nabla v_{1} \cdot \nabla v_{2} d x$. The discrete problem is again given by $(5.7)$.

The interpolation operator $\Pi_{k}: V \longrightarrow V_{k}$ is defined by the same formula in (5.8), and the estimate (5.10) remains valid (cf., [52]).

The operator $E_{k}: V_{k} \longrightarrow \tilde{V}_{k}$ is defined by $\left(E_{k} v\right)(m)=v(m)$ for all internal midpoints $m \in \mathcal{T}_{k},\left(E_{k} v\right)(c)=v(c)$ for all centroids $c \in \mathcal{T}_{k}$, and $\left(E_{k} v\right)(p)=$ average of $v_{i}(p)$ for all internal vertices $p \in \mathcal{T}_{k}$, where $v_{i}=\left.v\right|_{\mathrm{R}_{i}}$ and $\mathrm{R}_{i} \in \mathcal{T}_{k}$ contains $p$ as a vertex.

The operator $F_{k}: \tilde{V}_{k} \longrightarrow V_{k}$ is defined by the same formula in (5.12), and the intergrid transfer operator is defined by averaging as in Example 5.1.

All the assumptions in Section 2 can be verified for this example by the same arguments used in Example 5.1. Hence the results in Section 4 are applicable to the multigrid methods for (5.7) using the "rotated" $\mathcal{Q}_{1}$ finite elements.

Remark. The nonconforming $\mathcal{P}_{1}$ and "rotated" $\mathcal{Q}_{1}$ finite elements are equivalent to the lowest order triangular and rectangular Raviart-Thomas mixed finite elements (cf., [53], [4], [2]). There are multigrid methods for (5.1) using the lowest order Raviart-Thomas elements (cf., [19], [2]) which are based on the multigrid methods for the nonconforming elements. The results in Section 4 are therefore applicable to these multigrid algorithms for the lowest order Raviart-Thomas finite elements.

\section{Applications to a model fourth order problem}

In this section we apply our theory to the biharmonic equation with homogeneous Dirichlet boundary conditions. Let $\Omega$ be a bounded polygonal domain in $\mathbb{R}^{2}$ and 
$f \in L^{2}(\Omega)$. Consider the following boundary value problem.

$$
\Delta^{2} u=f \quad \text { in } \Omega \quad \text { and } \quad u=\frac{\partial u}{\partial n}=0 \quad \text { on } \partial \Omega .
$$

Let $V=H_{0}^{2}(\Omega)$, and let $a(\cdot, \cdot)$ on $V$ be defined by either

$$
a(v, w)=\sum_{i, j=1,2} \int_{\Omega} v_{x_{i} x_{j}} w_{x_{i} x_{j}} d x
$$

or

$$
a(v, w)=\int_{\Omega}\left[\Delta v \Delta w+(1-\sigma)\left(2 v_{x_{1} x_{2}} w_{x_{1} x_{2}}-v_{x_{1} x_{1}} w_{x_{2} x_{2}}-v_{x_{2} x_{2}} w_{x_{1} x_{1}}\right)\right] d x,
$$

where $\sigma$ is the Poisson ratio and $0<\sigma<\frac{1}{2}$. For either choice of the variational form $a(\cdot, \cdot)$, conditions (B) and (C) follow from the Cauchy-Schwarz inequality and the generalized Poincaré inequality (cf., [48]), respectively.

The weak formulation of (6.1) is to find $u \in V$ such that

$$
a(u, v)=\int_{\Omega} f v d x \quad \forall v \in V .
$$

By the elliptic regularity theory for non-smooth domains (cf., [36], [39], [40], [41]), there exists $\alpha \in\left(\frac{1}{2}, 1\right]$ such that for $f \in H^{-2+\alpha}(\Omega)$, the solution $u$ of $(6.2)$ belongs to $H^{2+\alpha}(\Omega)$ and

$$
\|u\|_{H^{2+\alpha}(\Omega)} \lesssim\|f\|_{H^{-2+\alpha}(\Omega)} .
$$

Let $Z=H^{2+\alpha}(\Omega) \cap H_{0}^{2}(\Omega)$ and $W=H_{0}^{2-\alpha}(\Omega)$. Clearly, (R-1) holds. Since $W^{\prime}=H^{-2+\alpha}(\Omega)$, the estimate (R-2) follows from (6.3).

Let $X=L^{2}(\Omega)$. By the interpolation of Sobolev spaces (cf., [61], [57]), we have

$$
Z=\left[H_{0}^{2}(\Omega), H^{3}(\Omega) \cap H_{0}^{2}(\Omega)\right]_{\alpha},
$$

and $W=\left[L^{2}(\Omega), H_{0}^{2}(\Omega)\right]_{1-\alpha / 2}$. In particular, the condition (C-3) holds.

The biharmonic operator $\Delta^{2}$ is a bounded linear operator from $H^{3}(\Omega)$ to $H^{-1}(\Omega)$, and from $H^{2}(\Omega)$ to $H^{-2}(\Omega)$. Therefore by interpolation we have

$$
\left\|\Delta^{2} \zeta\right\|_{H^{-2+\alpha}(\Omega)} \lesssim\|\zeta\|_{H^{2+\alpha}(\Omega)} \quad \forall \zeta \in H^{2+\alpha}(\Omega) .
$$

As in the case of the Poisson equation (cf., Section 5), the duality estimate (D) follows from (6.5) and a density argument.

We now consider finite element multigrid methods for (6.2). In the following examples, $\left\{\mathcal{T}_{k}\right\}_{k=1}^{\infty}$ is a sequence of quasi-uniform triangulations of $\Omega$. For simplicity we assume that $\mathcal{T}_{k+1}$ is obtained by connecting the midpoints of the edges of the triangles in $\mathcal{T}_{k}$. Let $T \in \mathcal{T}_{k}$. We denote by $S_{T}$ the interior of the union of the closures of the triangles in $\mathcal{T}_{k}$ neighboring $T$.

Example 6.1. Let $V_{k}^{*}=\left\{v \in L^{2}(\Omega):\left.v\right|_{T}\right.$ is quadratic, $v$ is continuous at the vertices and $\partial v / \partial n$ is continuous at the midpoints of interelement boundaries be the Morley finite element space associated with $\mathcal{T}_{k}$ (cf., [47]), and let $\tilde{V}_{k}^{*}$ be the Hsieh-Clough-Tocher macro element space associated with $\mathcal{T}_{k}$ (cf., [34]). A function $\tilde{v} \in \tilde{V}_{k}^{*}$ is $C^{1}$ on $\bar{\Omega}$, and its restriction to each $T \in \mathcal{T}_{k}$ is piecewise cubic on the three triangles formed by the centroid and the vertices of $T$. The space $V_{k}$ (resp., $\tilde{V}_{k}$ ) is the subspace of $V_{k}^{*}$ (resp., $\tilde{V}_{k}^{*}$ ) whose members have zero nodal values along $\partial \Omega$. Note that $V_{k} \preceq \tilde{V}_{k}$. The inner product for $V_{k}^{*}$ is defined by 
$(v, w)_{k}=h_{k}^{2} \sum_{p} v(p) w(p)+h_{k}^{4} \sum_{m} \frac{\partial v}{\partial n}(m) \frac{\partial w}{\partial n}(m)$, where the summations are taken over all vertices $p$ and midpoints $m$ in $\mathcal{T}_{k}$.

The symmetric positive definite bilinear form $a_{k}(\cdot, \cdot)$ is defined by either

$$
a_{k}(v, w)=\sum_{T \in \mathcal{T}_{k}} \sum_{i, j=1,2} \int_{T} v_{x_{i} x_{j}} w_{x_{i} x_{j}} d x
$$

or

$$
\begin{aligned}
a_{k}(v, w)=\sum_{T \in \mathcal{T}_{k}} \int_{T}[\Delta v \Delta & w+(1-\sigma) \\
\times & \left.\left(2 v_{x_{1} x_{2}} w_{x_{1} x_{2}}-v_{x_{1} x_{1}} w_{x_{2} x_{2}}-v_{x_{2} x_{2}} w_{x_{1} x_{1}}\right)\right] d x .
\end{aligned}
$$

The discrete problem for (6.2) is to find $u_{k} \in V_{k}$ such that

$$
a_{k}\left(u_{k}, v\right)=\int_{\Omega} f v d x \quad \forall v \in V_{k} .
$$

Clearly, (M), (C-1), (C-2), (I) and (P) are satisfied.

The interpolation operator $\Pi_{k}: V \longrightarrow V_{k}$ is defined by

$$
\left(\Pi_{k} v\right)(p)=v(p) \quad \text { and } \quad \frac{\partial\left(\Pi_{k} v\right)}{\partial n}(m)=\frac{1}{|e|} \int_{e} \frac{\partial v}{\partial n} d s,
$$

where $p$ and $m$ range over the internal vertices and midpoints of $\mathcal{T}_{k}$, and $m$ is the midpoint of the edge $e$. Note that

$$
\left.\left(\Pi_{k} \zeta\right)\right|_{T}=\left.\zeta\right|_{T} \quad \text { if }\left.\zeta\right|_{T} \text { is quadratic. }
$$

The following interpolation estimates are established by the standard techniques for almost affine family of finite elements (cf., [30]).

$$
\begin{aligned}
\left\|\zeta-\Pi_{k} \zeta\right\|_{L^{2}(T)} & +h_{k}\left|\zeta-\Pi_{k} \zeta\right|_{H^{1}(T)} \\
& +h_{k}^{2}\left|\zeta-\Pi_{k} \zeta\right|_{H^{2}(T)} \lesssim h_{k}^{\beta}|\zeta|_{H^{\beta}(T)}, \quad \beta=2,3,
\end{aligned}
$$

for all $T \in \mathcal{T}_{k}$ and $\zeta \in H^{\beta}(\Omega) \cap H_{0}^{2}(\Omega)$. The estimate (П-1) follows from (6.9) with $\beta=2$, and the estimate (П-2) follows from (П-1), (6.4), (6.9) with $\beta=3$ and interpolation. From (6.9) with $\beta=3$ we also have

$$
\left(\sum_{T \in \mathcal{T}_{k}}\left|\zeta-\Pi_{k} \zeta\right|_{H^{1}(T)}^{2}\right)^{1 / 2} \lesssim h_{k}^{2}\|\zeta\|_{H^{3}(\Omega)} \quad \forall \zeta \in H^{3}(\Omega) \cap H_{0}^{2}(\Omega) .
$$

Let $p$ and $m$ be the internal vertices and midpoints of $\mathcal{T}_{k}$. The operators $E_{k}$ : $V_{k} \longrightarrow \tilde{V}_{k}$ and $F_{k}: \tilde{V}_{k} \longrightarrow V_{k}$ are defined by

$$
\begin{aligned}
\left(E_{k} v\right)(p) & =v(p), \\
\frac{\partial\left(E_{k} v\right)}{\partial n}(m) & =\frac{\partial v}{\partial n}(m), \\
{\left[\partial^{\beta}\left(E_{k} v\right)\right](p) } & =\text { average of }\left(\partial^{\beta} v_{i}\right)(p), \quad|\beta|=1,
\end{aligned}
$$

where $v_{i}=\left.v\right|_{T_{i}}$ and $T_{i}$ contains $p$ as a vertex, and

$$
\left(F_{k} \tilde{v}\right)(p)=\tilde{v}(p) \quad \text { and } \quad \frac{\partial\left(F_{k} \tilde{v}\right)}{\partial n}(m)=\frac{\partial \tilde{v}}{\partial n}(m) .
$$

Note that $F_{k}$ is well-defined because $V_{k} \preceq \tilde{V}_{k}$. Clearly the relation (FE) holds, and (F) follows from a simple element by element calculation. 
Let $E_{k}^{*}: V_{k}^{*} \longrightarrow \tilde{V}_{k}^{*}$ be defined by the same formulas in (6.11) for all vertices and midpoints of $\mathcal{T}_{k}$. A straightforward computation (cf., the similar computation in [24] where the Argyris element was used instead of the Hsieh-Clough-Tocher element) yields

$$
\left\|E_{k}^{*} v-v\right\|_{L^{2}(T)}^{2} \lesssim h_{k}^{4} \sum_{K \in S_{T}}|v|_{H^{2}(K)}^{2} \quad \forall v \in V_{k}^{*}
$$

Moreover,

$$
\left.\left(E_{k}^{*} \eta\right)\right|_{T}=\eta \quad \text { if }\left.\eta\right|_{S_{T}} \text { is quadratic. }
$$

It follows that

$$
\left\|E_{k}^{*} v-v\right\|_{L^{2}(\Omega)}^{2} \lesssim h_{k}^{4} \sum_{T \in \mathcal{T}_{k}}|v|_{H^{2}(T)}^{2} \quad \forall v \in V_{k}^{*} .
$$

Let $v \in V_{k}$. Since $E_{k} v$ and $E_{k}^{*} v$ differ only by their first order derivatives at the vertices along $\partial \Omega$, we have

$$
\left\|E_{k} v-E_{k}^{*} v\right\|_{L^{2}(\Omega)}^{2} \lesssim h_{k}^{6} \Sigma^{\prime}\left\|\nabla^{2} v\right\|_{L^{\infty}(T)}^{2} \lesssim h_{k}^{4}\|v\|_{k}^{2} \quad \forall v \in V_{k},
$$

where the summation $\sum^{\prime}$ in (6.16) is taken over the triangles in $\mathcal{T}_{k}$ neighboring $\partial \Omega$.

The estimate (E) follows from (6.15) and (6.16). A standard inverse estimate then yields

$$
\left(\sum_{T \in \mathcal{T}_{k}}\left|E_{k} v-v\right|_{H^{1}(T)}^{2}\right)^{1 / 2} \lesssim h_{k}\|v\|_{k} \quad \forall v \in V_{k} .
$$

Using (6.8), (6.9), (6.13), (6.14) and the Bramble-Hilbert lemma as in Example 5.1 , we obtain

$$
\left\|E_{k}^{*} \Pi_{k} \zeta-\zeta\right\|_{L^{2}(\Omega)} \lesssim h_{k}^{3}|\zeta|_{H^{3}(\Omega)} \quad \forall \zeta \in H^{3}(\Omega) \cap H_{0}^{2}(\Omega) .
$$

Since $E_{k} \Pi_{k} \zeta$ and $E_{k}^{*} \Pi_{k} \zeta$ differ only by their first order derivatives at the vertices along $\partial \Omega$, we have, by (6.9),

$$
\begin{aligned}
\left\|E_{k} \Pi_{k} \zeta-E_{k}^{*} \Pi_{k} \zeta\right\|_{L^{2}(\Omega)}^{2} & \left.\lesssim h_{k}^{4} \sum_{p \in \partial \Omega} \sum_{\substack{T \in \mathcal{T}_{k} \\
\bar{T} \ni p}}\left|\nabla\left(\Pi_{k} \zeta\right)\right|_{T}\right|^{2}(p) \\
& \lesssim h_{k}^{4} \sum_{p \in \partial \Omega} \sum_{\substack{T \in \mathcal{T}_{k} \\
\bar{T} \ni p}}\left|\nabla\left(\Pi_{k} \zeta\right)\right|_{T}-\left.\nabla \zeta\right|^{2}(p) \\
& \lesssim h_{k}^{6}|\zeta|_{H^{3}(\Omega)}^{2} \quad \forall \zeta \in H^{3}(\Omega) \cap H_{0}^{2}(\Omega) .
\end{aligned}
$$

It follows from (6.18) and (6.19) that

$$
\left\|E_{k} \Pi_{k} \zeta-\zeta\right\|_{L^{2}(\Omega)} \lesssim h_{k}^{3}|\zeta|_{H^{3}(\Omega)} \quad \forall \zeta \in H^{3}(\Omega) \cap H_{0}^{2}(\Omega) .
$$

By (6.9), (6.20) and standard inverse estimates, we have

$$
\begin{aligned}
\left\|E_{k} \Pi_{k} \zeta-\zeta\right\|_{H^{2}(\Omega)} & \lesssim h_{k}^{-2}\left\|E_{k} \Pi_{k} \zeta-\Pi_{k} \zeta\right\|_{L^{2}(\Omega)}+\left\|\Pi_{k} \zeta-\zeta\right\|_{H^{2}(\Omega)} \\
& \lesssim h_{k}|\zeta|_{H^{3}(\Omega)} \quad \forall \zeta \in H^{3}(\Omega) \cap H_{0}^{2}(\Omega) .
\end{aligned}
$$

By (I), (E) and (П-1) we also have the trivial estimate (6.22) $\left\|E_{k} \Pi_{k} \zeta-\zeta\right\|_{L^{2}(\Omega)}+h_{k}^{2}\left\|E_{k} \Pi_{k} \zeta-\zeta\right\|_{H^{2}(\Omega)} \lesssim h_{k}^{2}\|\zeta\|_{H^{2}(\Omega)} \quad \forall \zeta \in H_{0}^{2}(\Omega)$.

The estimate (ЕП) follows from (6.4), (6.20), (6.21), (6.22) and interpolation. 
Next we turn to the assumptions $(\mathrm{N}-1)$ and $(\mathrm{N}-2)$. Let $\zeta \in H^{3}(\Omega) \cap H_{0}^{2}(\Omega)$ and $\zeta_{k} \in V_{k}$ be related to $\zeta$ through (2.4). Let $v \in V_{k}+V$; then by the Green's formula (cf., [59]) we have

$$
a_{k}(\zeta, v)=-\sum_{T \in \mathcal{T}_{k}} \int_{T} \nabla(\Delta \zeta) \cdot \nabla v d x+\sum_{e} \int_{e}\left(G_{1}(\zeta)\left[v_{x_{1}}\right]+G_{2}(\zeta)\left[v_{x_{2}}\right]\right) d s
$$

where $G_{1}(\zeta)$ and $G_{2}(\zeta)$ are combinations of second order derivatives of $\zeta,\left[v_{x_{1}}\right]$ and $\left[v_{x_{2}}\right]$ denote the jumps of $v_{x_{1}}$ and $v_{x_{2}}$ across the edge $e$, and the second summation is taken over all edges $e$ of $\mathcal{T}_{k}$.

Since $E_{k} v \in H_{0}^{2}(\Omega)$, it follows from (2.4) and (6.23) that

$$
a_{k}\left(\zeta_{k}, v\right)=-\sum_{T \in \mathcal{T}_{k}} \int_{T} \nabla(\Delta \zeta) \cdot \nabla\left(E_{k} v\right) d x
$$

By subtracting (6.24) from (6.23) we obtain

$$
\begin{aligned}
a_{k}\left(\zeta-\zeta_{k}, v\right)= & -\sum_{T \in \mathcal{T}_{k}} \int_{T} \nabla(\Delta \zeta) \cdot \nabla\left(v-E_{k} v\right) d x \\
& +\sum_{e} \int_{e}\left(G_{1}(\zeta)\left[v_{x_{1}}\right]+G_{2}(\zeta)\left[v_{x_{2}}\right]\right) d s \quad \forall v \in V_{k} .
\end{aligned}
$$

By the Cauchy-Schwarz inequality and (6.17) we have

$$
\left|\sum_{T \in \mathcal{T}_{k}} \int_{T} \nabla(\Delta \zeta) \cdot \nabla\left(v-E_{k} v\right) d x\right| \lesssim h_{k}\|\zeta\|_{H^{3}(\Omega)}\|v\|_{k} \quad \forall v \in V_{k} .
$$

Since $v_{x_{1}}$ and $v_{x_{2}}$ are continuous at the midpoints, we have, by a standard argument for nonconforming finite elements,

$$
\left|\sum_{e} \int_{e}\left(G_{1}(\zeta)\left[v_{x_{1}}\right]+G_{2}(\zeta)\left[v_{x_{2}}\right]\right) d s\right| \lesssim h_{k}\|\zeta\|_{H^{3}(\Omega)}\|v\|_{k} \quad \forall v \in V_{k} .
$$

Combining (6.25)-(6.27), we obtain

$$
\left|a_{k}\left(\zeta-\zeta_{k}, v\right)\right| \lesssim h_{k}\|\zeta\|_{H^{3}(\Omega)}\|v\|_{k} \quad \forall v \in V_{k}
$$

Let $\zeta \in H_{0}^{2}(\Omega)$. Then we have the obvious estimate

$$
\left\|\zeta_{k}\right\|_{k} \lesssim\|\zeta\|_{H^{2}(\Omega)}
$$

which implies that

$$
\left|a_{k}\left(\zeta-\zeta_{k}, v\right)\right| \lesssim\left\|\zeta-\zeta_{k}\right\|_{k}\|v\|_{k} \lesssim\|\zeta\|_{H^{2}(\Omega)}\|v\|_{k} \quad \forall v \in V_{k}
$$

The estimate (N-1) now follows from (6.4), (6.28), (6.30) and interpolation.

By (6.9) and (6.20) we have

$$
\begin{aligned}
\left\|E_{k} \Pi_{k} \xi-\Pi_{k} \xi\right\|_{L^{2}(\Omega)} & \lesssim\left\|E_{k} \Pi_{k} \xi-\xi\right\|_{L^{2}(\Omega)}+\left\|\xi-\Pi_{k} \xi\right\|_{L^{2}(\Omega)} \\
& \lesssim h_{k}^{3}\|\xi\|_{H^{3}(\Omega)} \quad \forall \xi \in H^{3}(\Omega) \cap H_{0}^{2}(\Omega) .
\end{aligned}
$$

A standard inverse estimate then implies that

$$
\left(\sum_{T \in \mathcal{T}_{k}}\left|E_{k} \Pi_{k} \xi-\Pi_{k} \xi\right|_{H^{1}(T)}^{2}\right)^{1 / 2} \lesssim h_{k}^{2}\|\xi\|_{H^{3}(\Omega)} \quad \forall \xi \in H^{3}(\Omega) \cap H_{0}^{2}(\Omega) .
$$


It follows from (6.25) that

$$
\begin{aligned}
a_{k}(\zeta & \left.-\zeta_{k}, \Pi_{k} \xi\right)=-\sum_{T \in \mathcal{T}_{k}} \int_{T} \nabla(\Delta \zeta) \cdot \nabla\left(\Pi_{k} \xi-E_{k} \Pi_{k} \xi\right) d x \\
& +\sum_{e} \int_{e}\left(G_{1}(\zeta)\left[\left(\Pi_{k} \xi\right)_{x_{1}}\right]+G_{2}(\zeta)\left[\left(\Pi_{k} \xi\right)_{x_{2}}\right]\right) d s
\end{aligned}
$$

for $\zeta, \xi \in H^{3}(\Omega) \cap H_{0}^{2}(\Omega)$.

By the Cauchy-Schwarz inequality and (6.32) we have

$$
\left|\sum_{T \in \mathcal{T}_{k}} \int_{T} \nabla(\Delta \zeta) \cdot \nabla\left(\Pi_{k} \xi-E_{k} \Pi_{k} \xi\right) d x\right| \lesssim h_{k}^{2}\|\zeta\|_{H^{3}(\Omega)}\|\xi\|_{H^{3}(\Omega)}
$$

for $\zeta, \xi \in H^{3}(\Omega) \cap H_{0}^{2}(\Omega)$.

A standard argument for nonconforming finite elements shows that

$$
\begin{aligned}
& \left|\sum_{e} \int_{e}\left(G_{1}(\zeta)\left[\left(\Pi_{k} \xi\right)_{x_{1}}\right]+G_{2}(\zeta)\left[\left(\Pi_{k} \xi\right)_{x_{2}}\right]\right) d s\right| \\
& \quad \lesssim\left|\sum_{e} \int_{e}\left(G_{1}(\zeta)\left[\left(\Pi_{k} \xi\right)_{x_{1}}-\xi_{x_{1}}\right]+G_{2}(\zeta)\left[\left(\Pi_{k} \xi\right)_{x_{2}}-\xi_{x_{2}}\right]\right) d s\right| \\
& \quad \lesssim h_{k}^{2}\|\zeta\|_{H^{3}(\Omega)}\|\xi\|_{H^{3}(\Omega)} \quad \forall \zeta, \xi \in H^{3}(\Omega) \cap H_{0}^{2}(\Omega) .
\end{aligned}
$$

Combining (6.33)-(6.35), we obtain

$$
\left|a_{k}\left(\zeta-\zeta_{k}, \Pi_{k} \xi\right)\right| \lesssim h_{k}^{2}\|\zeta\|_{H^{3}(\Omega)}\|\xi\|_{H^{3}(\Omega)} \quad \forall \zeta, \xi \in H^{3}(\Omega) \cap H_{0}^{2}(\Omega) .
$$

On the other hand, for $\zeta, \xi \in H_{0}^{2}(\Omega)$, the estimates (6.9) and (6.29) imply that

$$
\left|a_{k}\left(\zeta-\zeta_{k}, \Pi_{k} \xi\right)\right| \lesssim\|\zeta\|_{H^{2}(\Omega)}\|\xi\|_{H^{2}(\Omega)} .
$$

The estimate (N-2) now follows from (6.4), (6.36), (6.37) and (bilinear) interpolation.

The intergrid transfer operator $I_{k-1}^{k}: V_{k-1} \longrightarrow V_{k}$ is defined by averaging as follows. Let $p$ be a vertex of $\mathcal{T}_{k}$ inside $\Omega$. If $p$ is also a vertex of $\mathcal{T}_{k-1}$, then $\left(I_{k-1}^{k} v\right)(p)=v(p)$. If $p$ is the midpoint of the common edge of two triangles $T_{1}$ and $T_{2} \in \mathcal{T}_{k-1}$, then

$$
\left(I_{k-1}^{k} v\right)(p)=\frac{1}{2}\left[\left.v\right|_{T_{1}}(p)+\left.v\right|_{T_{2}}(p)\right] .
$$

Let $m$ be a midpoint of an edge $e$ of $\mathcal{T}_{k}$ inside $\Omega$ and $n$ be a unit normal of $e$. If $m$ is in the interior of a triangle in $\mathcal{T}_{k-1}$, then

$$
\frac{\partial\left(I_{k-1}^{k} v\right)}{\partial n}(m)=\frac{\partial v}{\partial n}(m)
$$

If $m$ is on the common edge of two triangles $T_{1}$ and $T_{2}$ in $\mathcal{T}_{k-1}$, then

$$
\frac{\partial\left(I_{k-1}^{k} v\right)}{\partial n}(m)=\frac{1}{2}\left[\frac{\left.\partial v\right|_{T_{1}}}{\partial n}(m)+\frac{\left.\partial v\right|_{T_{2}}}{\partial n}(m)\right] .
$$

The estimate (I-1) follows immediately from the estimates in [16], and the estimate (I-2) follows from the estimates in [16] and interpolation, as in Example 5.1.

Since all of the assumptions of our theory hold for this example, the results in Section 4 are applicable to the multigrid methods for (6.6). 
Remark. For $1 / 2<\alpha<1$, the estimate (4.31) and the Sobolev inequality (cf., [61]) imply that

$$
\sup _{x \in \bar{\Omega}}\left|u(x)-\left[E_{k} \hat{u}_{k}\right](x)\right| \lesssim h_{k}^{2 \alpha}\|u\|_{H^{2+\alpha}(\Omega)}+h_{k}^{2+\alpha}\|f\|_{L^{2}(\Omega)} .
$$

Since $E_{k} v$ and $v$ coincide at the vertices, we have

$$
\max _{p}\left|u(p)-\hat{u}_{k}(p)\right| \lesssim h_{k}^{2 \alpha}\|u\|_{H^{2+\alpha}(\Omega)}+h_{k}^{2+\alpha}\|f\|_{L^{2}(\Omega)},
$$

where the summation is taken over all the vertices of $\mathcal{T}_{k}$.

In the case $\alpha=1$, we have, for any $0<\beta<1$,

$$
\max _{p}\left|u(p)-\hat{u}_{k}(p)\right| \leq C_{\beta}\left[h_{k}^{2 \beta}\|u\|_{H^{2+\beta}(\Omega)}+h_{k}^{2+\beta}\|f\|_{L^{2}(\Omega)}\right] .
$$

Remark. The symmetric variable $V$-cycle preconditioner for the Morley finite element method can also be used to precondition the Argyris finite element method (cf., [3], [26]).

Remark. The results in Example 6.1 are also valid for the Adini element (cf., [1], [29], [45]) and the incomplete biquadratic element (cf., [58]), which are connected to the Bogner-Fox-Schmit element (cf., [8]) and the Fraeijs de Veubeke-Sander quadrilateral element (cf., [54], [38], [32]), respectively.

Example 6.2. Let $V_{k}=\tilde{V}_{k} \subseteq H_{0}^{2}(\Omega)$ be the Hsieh-Clough-Tocher or the reduced Hsieh-Clough-Tocher macro finite element space associated with $\mathcal{T}_{k}$ (cf., [34], [31], [51]), and let $a_{k}(\cdot, \cdot)=a(\cdot, \cdot)$ on $V_{k}$. The discrete problem for $(6.2)$ is to find $u_{k} \in V_{k}$ such that

$$
a\left(u_{k}, v\right)=\int_{\Omega} f v d x \quad \forall v \in V_{k} .
$$

There exists an interpolation operator $\Pi_{k}: V \longrightarrow V_{k}$ such that

$$
\left\|\zeta-\Pi_{k} \zeta\right\|_{L^{2}(T)}+h_{k}^{2}\left|\zeta-\Pi_{k} \zeta\right|_{H^{2}(T)} \lesssim h_{k}^{\beta}|\zeta|_{H^{\beta}\left(S_{T}\right)}, \quad \beta=2,3,
$$

and

$$
\left.\left(\Pi_{k} \zeta\right)\right|_{T}=\left.\zeta\right|_{T} \quad \text { if }\left.\zeta\right|_{S_{T}} \text { is quadratic. }
$$

The estimates ( $\Pi-1)$ and ( -2$)$ follow from (6.40). The operator $\Pi_{k}$ can be constructed by using the techniques in [33] and [56]. For the Hsieh-Clough-Tocher element, we can also take $\Pi_{k}$ to be the composition of the interpolation operator for the Morley element defined in (6.7) and the connection operator defined in (6.11).

Let $E_{k}=F_{k}=$ identity map on $V_{k}$. The estimates (E), (Eח), (F), (FE), (N-1) and $(\mathrm{N}-2)$ are then completely trivial. We can take $I_{k-1}^{k}: V_{k-1} \longrightarrow V_{k}$ to be the nodal interpolation operator. Then (I-1) is a standard interpolation error estimate. Moreover, we have

$$
\left.\left(I_{k-1}^{k} v\right)\right|_{T}=\left.v\right|_{T} \quad \text { if }\left.v\right|_{T} \text { is quadratic. }
$$

The estimates (П-1) and (I-1) imply that

$$
\left\|I_{k-1}^{k} \Pi_{k-1} \zeta-\Pi_{k} \zeta\right\|_{L^{2}(\Omega)} \lesssim h_{k}^{2}\|\zeta\|_{H^{2}(\Omega)} \quad \forall \zeta \in H_{0}^{2}(\Omega) .
$$


Using (6.41), (6.42) and the Bramble-Hilbert lemma, we obtain (cf., the proof of $(6.20))$

$$
\left\|I_{k-1}^{k} \Pi_{k-1} \zeta-\Pi_{k} \zeta\right\|_{L^{2}(\Omega)} \lesssim h_{k}^{3}\|\zeta\|_{H^{3}(\Omega)} \quad \forall \zeta \in H^{3}(\Omega) \cap H_{0}^{2}(\Omega)
$$

The estimate (I-2) follows from (6.4), (6.43), (6.44) and interpolation.

Therefore the results from Section 4 can be applied to these macro element methods.

\section{REFERENCES}

1. A. Adini and R.W. Clough, Analysis of plate bending by the finite element method, NSF Report G. 7337 (1961).

2. T. Arbogast and Z. Chen, On the implementation of mixed methods as nonconforming methods for second order elliptic problems, Math. Comp. 64 (1995), 943-972.

3. J.H. Argyris, I. Fried and D.W. Scharpf, The TUBA family of plate elements for the matrix displacement method, Aero. J. Roy. Aero. Soc. 72 (1968), 701-709.

4. D.N. Arnold and F. Brezzi, Mixed and nonconforming finite element methods: implementation, postprocessing and error estimates, R.A.I.R.O Modél. Math. Anal. Numér. 19 (1985), 7-32. MR 87g:65126

5. D.N. Arnold and R.S. Falk, A uniformly accurate finite element method for the ReissnerMindlin plate, SIAM J. Numer. Anal. 21 (1990), 281-312. MR 91c:65068

6. R.E. Bank and T.F. Dupont, An optimal order process for solving finite element equations, Math. Comp. 36 (1981), 35-51. MR 82b:65113

7. J. Bergh and J. Löfström, Interpolation Spaces, Springer-Verlag, Berlin, 1976. MR 58:2349

8. F.K. Bogner, R.L. Fox and L.A. Schmit, The generation of interelement compatible stiffness and mass matrices by the use of interpolation formulas, Proc. Conf. Matrix Methods in Structural Mechanics (1965).

9. D. Braess and R. Verfürth, Multigrid methods for nonconforming finite element methods, SIAM J. Numer. Anal. 27 (1990), 979-986. MR 95j:65164

10. J.H. Bramble, Multigrid Methods, Longman Scientific \& Technical, Essex, 1993. MR 95b:65002

11. J.H. Bramble and S.R. Hilbert, Estimation of linear functionals on Sobolev spaces with application to Fourier transforms and spline interpolation, SIAM J. Numer. Anal. 7 (1970), 113-124. MR 41:7819

12. J.H. Bramble and J.E. Pasciak, New estimates for multigrid algorithms including the $V$-cycle, Math. Comp. 60 (1993), 447-471. MR 94a:65064

13. J.H. Bramble, J.E. Pasciak and J. Xu, The analysis of multigrid algorithms with nonnested spaces or noninherited quadratic forms, Math. Comp. 56 (1991), 1-34. MR 91h:65159

14. S.C. Brenner, Multigrid Methods for Nonconforming Finite Elements, Dissertation, The University of Michigan, 1988.

15. _ An optimal-order multigrid method for $P 1$ nonconforming finite elements, Math. Comp. 52 (1989), 1-15. MR 89f:65119

16. _ An optimal-order nonconforming multigrid method for the biharmonic equation, SIAM J. Numer. Anal. 26 (1989), 1124-1138. MR 90i:65189

17. _ Multigrid methods for nonconforming finite elements, Copper Mountain Conference on Multigrid methods (J. Mandel, et. al., eds.), SIAM, Philadelphia, 1989, pp. 54-65. MR 91h:65189

18. _ A nonconforming multigrid method for the stationary Stokes equations, Math. Comp. 52 (1990), 411-437. MR 91d:65167

19. __ A multigrid algorithm for the lowest-order Raviart-Thomas mixed triangular finite element method, SIAM J. Numer. Anal. 29 (1992), 647-678. MR 93j:65175

20. _ A nonconforming mixed multigrid method for the pure displacement problem in planar linear elasticity, SIAM J. Numer. Anal. 30 (1993), 116-135. MR 93m:65166 
21. _ A nonconforming mixed multigrid method for the pure traction problem in planar linear elasticity, Math. Comp. 63 (1994), 435-460, S1-S5. MR 95c:73076

22. — Two-level additive Schwarz preconditioners for nonconforming finite elements, Domain Decomposition Methods in Scientific and Engineering Computing (D. Keyes and J. $\mathrm{Xu}$, eds.), Proceedings of the Seventh International Conference on Domain Decomposition, Contemp. Math. 180, 1994, pp. 9-14. MR 95j:65134

23. _ Multigrid methods for parameter dependent problems, RAIRO Modél. Math. Anal. Numér. 30 (1996), 265-297. MR 97c:73076

24. Comp. 65 (1996), 897-921. MR 96j:65117

25. _ A two-level additive Schwarz preconditioner for nonconforming plate elements, $\mathrm{Nu}$ mer. Math. 72 (1996), 419-447. MR 97h:65147

26. _ Preconditioning complicated FEMs by simple FEMs, SIAM J. Sci. Comput. 17 (1996), 1269-1274. MR 97g:65226

27. S.C. Brenner and L.R. Scott, The Mathematical Theory of Finite Element Methods, SpringerVerlag, Berlin, 1994. MR 95f:65001

28. S.C. Brenner and L.-Y. Sung, Linear finite element methods for planar linear elasticity, Math. Comp. 59 (1992), 321-338. MR 93a:73078

29. P.G. Ciarlet, Conforming and nonconforming finite element methods for solving the plate problem, Conference on the Numerical Solution of Differential Equations (G.A. Watson, ed.), Lecture Notes in Mathematics, vol. 363, Springer-Verlag, Berlin, 1974, pp. 21-31. MR 54:11806

30. _ The Finite Element Method for Elliptic Problems, North-Holland, Amsterdam, 1978. MR 58:25001

31. _ Interpolation error estimates for the reduced Hsieh-Clough-Tocher triangle, Math. Comp. 32 (1978), 335-344. MR 80i:65114

32. J.F. Ciavaldini and J.C. Nedelec, Sur l'élément de Fraeijs de Veubeke et Sander, RAIRO R-2 (1974), 29-45. MR 52:2247

33. P. Clément, Approximation by finite element functions using local regularization, R.A.I.R.O. R-2 (1975), 77-84. MR 53:4569

34. R.W. Clough and J.L. Tocher, Finite element stiffness matrices for analysis of plates in bending, Proceedings of the Conference on Matrix Methods in Structural Mechanics, Wright Patterson A.F.B., Ohio, 1965.

35. M. Crouzeix and P.-A. Raviart, Conforming and nonconforming finite element methods for solving the stationary Stokes equations I, R.A.I.R.O. R-3 (1973), 33-75. MR 49:8401

36. M. Dauge, Elliptic Boundary Value Problems on Corner Domains, Lecture Notes in Math., vol. 1341, Springer-Verlag, Berlin, 1988. MR 91a:35078

37. R.S. Falk, Nonconforming finite element methods for the equations of linear elasticity, Math. Comp. 57 (1991), 529-550. MR 92a:65290

38. B. Fraeijs de Veubeke, A conforming finite element for plate bending, Int. J. Solids and Structure 4 (1968), 95-108.

39. P. Grisvard, Elliptic Problems in Nonsmooth Domains, Pitman, London, 1985. MR 86m:35044

40. __ Problèmes aux limites dans les polygones, Mode d'emploi, EDF Bull. Direction Études Rech. Sér. C. Math. Inform. 1 (1986), 21-59. MR 87g:35073

41. _ Singularities in Boundary Value Problems, Springer-Verlag, Berlin, 1992. MR 93h:35004

42. W. Hackbusch, Multigrid Methods and Applications, Springer-Verlag, Berlin, 1985. MR 87e: 65082

43. M.R. Hanisch, Multigrid preconditioning for the biharmonic Dirichlet problem, SIAM J. Numer. Anal. 30 (1993), 184-214. MR 94e:65131

44. S.G. KreĬn, Ju. I. Petunin and E.M. Semenov, Interpolation of Linear Operators, Translations of Mathematical Monographs, vol. 54, American Mathematical Society, Providence, 1982.

45. P. Lascaux and P. Lesaint, Some nonconforming finite elements for the plate bending problem, R.A.I.R.O. R-1 (1975), 9-53. MR 54:11941

46. S. McCormick (ed.), Multigrid Methods, SIAM Frontiers in Applied Mathematics 3, SIAM, Philadelphia, 1987. MR 89m:65004 
47. L.S.D. Morley, The triangular equilibrium problem in the solution of plate bending problems, Aero. Quart. 19 (1968), 149-169.

48. J. Nečas, Les Méthodes Directes en Théorie des Équations Elliptiques, Masson, Paris, 1967. MR 37:3168

49. P. Peisker and D. Braess, A conjugate gradient method and a multigrid algorithm for Morley's finite element approximation of the biharmonic equation, Numer. Math. 50 (1987), 567-586. MR 88e: 65147

50. P. Peisker, W. Rust and E. Stein, Iterative solution methods for plate bending problems: multigrid and preconditioned cg algorithm, SIAM J. Numer. Anal. 27 (1990), 1450-1465. MR 91h:73055

51. P. Percell, On cubic and quartic Clough-Tocher finite elements, SIAM J. Numer. Anal. 13 (1976), 100-103. MR 53:11963

52. R. Rannacher and S. Turek, Simple nonconforming quadrilateral Stokes element, Numer. Meth. PDE 8 (1992), 97-111. MR 92i:65170

53. P.-A. Raviart and J.M. Thomas, A mixed finite element method for second order elliptic problems, Mathematical Aspects of the Finite Element Method (I.Galligani and E. Magenes, eds.), Lecture Notes in Mathematics, vol. 606, Springer-Verlag, Berlin, 1977, pp. 292-315. MR 58:3547

54. G. Sander, Bornes supérieures et inférieures dans l'analyse matricielle des plaques en flexiontorsion, Bull. Soc. Roy. Sci. Liège 33 (1964), 456-494. MR 30:764

55. P. Schreiber and S. Turek, Multigrid results for the nonconforming Morley element, preprint (1993).

56. L.R. Scott and S. Zhang, Finite element interpolation of non-smooth functions satisfying boundary conditions, Math. Comp. 54 (1990), 483-493. MR 90j:65021

57. R. Seeley, Interpolation in $L^{p}$ with boundary conditions, Studia Math. 44 (1972), 47-60. MR 47:3981

58. Z. Shi, On the convergence of the incomplete biquadratic nonconforming plate element, Math. Numer. Sinica 8 (1986), 53-62 (Chinese). MR 87j:73084

59. _ Error estimates of Morley element, Chinese J. Num. Math. \& Appl. 12 (1990), 9-15. MR 91i:65182

60. Z. Shi, X. Yu and Z. Xie, A multigrid method for Bergan's energy-orthogonal plate element, Advances in Numerical Mathematics: Proc. Second Japan-China Sem. (Tokyo, 1994), Lecture Notes Numer. Appl. Anal., vol. 14, Kinokuniya, Tokyo, 1995, pp. 159-169. MR 98g:73041

61. H. Triebel, Interpolation Theory, Function Spaces, Differential Operators, North-Holland, Amsterdam, 1978. MR 80i:46032

62. S. Turek, Ein robustes und effizientes Mehrgitterverfahren zur Lösung der instationären, inkompressiblen 2-D Navier-Stokes-Gleichungen mit diskret divergenzfreien finiten Elementen, Dissertation, Universität Heidelberg, 1991.

63. _ Multigrid techniques for a divergence-free finite element discretization, East-West J. Numer. Math. 2 (1994), 229-255. MR 96c:65195

64. M. Wang, The multigrid method for TRUNC plate element, J. Comput. Math. 11 (1993), 178-187. MR 94m:65192

65. - The $W$-cycle multigrid method for finite elements with nonnested spaces, Adv. in Math. 23 (1994), 238-250. MR 95e:65118

66. S. Zhang, An optimal order multigrid method for biharmonic, $C^{1}$ finite element equations, Numer. Math. 56 (1989), 613-624. MR 90j:65135

67. Z. Zhang and S. Zhang, Wilson's element for the Reissner-Mindlin plate, Comput. Methods Appl. Mech. Engrg. 113 (1994), 55-65. MR 94k:73078

68. S. Zhou and G. Feng, Multigrid method for the Zienkiewicz element approximation of biharmonic equation, J. Hunan Univ. 20 (1993), 1-6 (Chinese). MR 94c:65155

Department of Mathematics, University of South Carolina, Columbia, SC 29208

E-mail address: brenner@math.sc.edu 